\title{
Three generations of granitoids emplaced over a 300 My time span in the Strzelin Massif, Fore-Sudetic Block, SW Poland: mutual relationships and implications for secular crustal evolution
}

\author{
Teresa OBERC-DZIEDZIC ${ }^{1 *}$, Christian PIN², Stanisław MADEJ ${ }^{1}$, Ryszard KRYZA ${ }^{1 \dagger}$ \\ ${ }^{1}$ Institute of Geological Sciences, University of Wrocław, 50-205 Wrocław, Plac M. Borna 9, Poland; teresa.oberc-dziedzic@ing.uni.wroc.pl \\ ${ }^{2}$ Département de Géologie, CNRS, Université Blaise Pascal, Campus des Cézeaux, 63178 Aubière Cedex, France \\ Deceased \\ ${ }^{*}$ Corresponding author
}

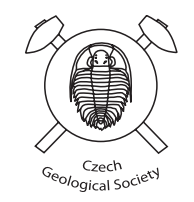

In the Sudetes, northeastern Bohemian Massif, three groups of (meta-)granitoids occur: 1) Cadomian orthogneisses, with a protolith $c .600-540 \mathrm{Ma}$ old, 2) Early Ordovician ( 500 Ma) orthogneisses, and finally 3) Variscan ( 300 Ma) granitoids. The three groups exist together in the Karkonosze-Izera Massif in the West Sudetes and in the Strzelin Massif in the East Sudetes.

In the Strzelin Massif, the $\sim 600$ Ma old granitoids belong to the Strzelin Complex ascribed to the Brunovistulian microcontinent, whereas the $\sim 500$ Ma orthogneisses are part of the allochtonous Stachów Complex, correlated with the Saxo-Thuringian Zone. During the Variscan Orogeny, the Stachów Complex was thrust onto the Strzelin Complex. Subsequently, both complexes were intruded by Variscan granitoids.

Trace-element data support the crustal affinity of the three generations of granitoids but they do not provide evidence for their mutual relationships. Neodymium isotope data suggest that, while the $600 \mathrm{Ma}$ orthogneisses were produced from ancient, purely crustal source materials, the genesis of the Ordovician metagranites involved protoliths that were isotopically less mature. Finally, Variscan granitoids required a significant contribution of juvenile (mantle-derived) components.

The general increase of initial $\varepsilon N d$ values with decreasing age is reflected by the younging average crustal residence ages of the source materials to each particular group of granitoids in the Strzelin Massif. Although they are allochtonous in the massif, the $500 \mathrm{Ma}$ gneisses fit very well to the scheme: the younger granites, the less negative values of the $\varepsilon \mathrm{Nd}_{i}$. The zircon inherited-core ages suggest that the protoliths of the $600 \mathrm{Ma}$ and $500 \mathrm{Ma}$ gneisses originated from different sources derived from Avalonia- and Armorica-type terranes, respectively.

Keywords: Strzelin Massif granitoids, continental crust, trace elements, Nd isotopes, $U-P b$ zircon ages, Sudetes

Received: 19 September, 2016; accepted: 12 January, 2017; handling editor: J. Žák

The online version of this article (doi: 10.3190/jgeosci.227) contains supplementary electronic material.

\section{Introduction}

The origin of the continental crust is one of the most important aspects of the evolution of the Earth as a planet and it has attracted much attention of geologists for many decades. Models of the formation and evolution of the continental crust are based on assumptions on its average composition, structure and age relationships. Particularly difficult problems concern the nature of the lower continental crust. Our knowledge of the lower crust mainly comes from: 1) deep-crustal xenoliths transported upwards by ascending magmas, 2) very high-grade metamorphic rocks (e.g. granulites) tectonically exhumed to the Earth's surface, and 3) granites, largely produced by partial melting at lower crustal depths (e.g. Kemp and Hawkesworth 2005).

The recently increasing amount of data obtained by various research methods provides new insights into complexities of the structure and processes operating in the Earth's crust, both on the global and regional scales. The regional aspect of the continental crust evolution can be well exemplified through a comparison of granites of different ages emplaced in a given crustal segment.

These features include, but are not restricted to:

1. major and trace elements that constrain sources of granite magmas as well as their subsequent differentiation (McLennan et al. 2006);

2. neodymium isotope characteristics of granites, metagranitoids and metasedimentary country rocks that provide information on the relative contributions of recycled, old continental crust and juvenile, mantle-derived components to the genesis of granitic magmas (Patchett and Samson 2005);

3. in situ $\mathrm{U}-\mathrm{Pb}$ ages of accessory zircon in granites, metagranitoids and country rocks constraining the timing of granite emplacement events and revealing pos- 
sible inheritance from crustal protoliths (e.g., Friedl et al. 2000).

By virtue of the abundance of granitoid rocks emplaced over a several hundred My time span, the European Variscan Belt offers a good place for such studies. At its north-eastern exposed edge, the Polish Sudetes, three generations of granitoids have been documented in the Karkonosze-Izera Massif of the west Sudetes and in the Strzelin Massif of the east Sudetes (Fig. 1).

The aim of this study is to compare the geochemistry of these three generations in the Strzelin Massif, in order to draw inferences on their sources and their possible relationships. This is based on published (granites) and partly published (tonalites) geochemical and isotopic characteristics, which are compared with unpublished data from to the neighboring Neoproterozoic and early Paleozoic metagranitoids. The whole-rock geochemical and $\mathrm{Nd}$ isotopic pieces of evidence on the mutual relationships of the three generations of granitoids are completed by published $\mathrm{U}-\mathrm{Pb}$ ages of inherited zircon cores that provide further information on the source regions of the parental granitic magmas.

\section{Geological setting}

Outside the Alpine realm, the crystalline basement units of the Variscan Belt of Europe (Suess 1888) crop out in isolated massifs from the Iberian Meseta in Portugal and Spain, through the Armorican Massif and Massif Central in France, the Vosges and Black Forest in France and Germany, to the Bohemian Massif in the east (Fig. 1a).

The Bohemian Massif comprises the Moldanubian, Saxo-Thuringian (Kossmat 1927) and Teplá-Barrandian (Franke 1989) zones, interpreted as terranes (Matte et al. 1990; Franke and Żelaźniewicz 2000) of the Armorican Terrane Assemblage (Franke 2000) and the western margin of the Brunovistulicum (Dudek 1980) interpreted as a microcontinent (Pharaoh 1999; Schulmann and Gayer 2000) of Avalonian affinity (Finger et al. 2000; Friedl et al. 2000). Both parts adjoin along the NNE-SSWtrending Moldanubian overthrust (Suess 1912, 1926).

The Sudetes situated in the northeasternmost part of the Bohemian Massif (Fig. 1b) are bordered by the WNW-ESE-trending Elbe Fault Zone in the SW and the Middle Odra Fault Zone in the NE. They are subdivided into the mountainous Sudetic Block to the SW and the largely peneplained Fore-Sudetic Block to the NE, separated by the NW-SE-trending Sudetic Marginal Fault (Fig. 1c). In a terrane perspective, the Sudetes can be subdivided into the West and Central Sudetes (Mazur et al. 2006), which belong to the "Armorican" part of the Bohemian Massif, and the East Sudetes that are part of the Brunovistulicum.

\subsection{Granitoids in the Sudetes}

The Sudetes abound in granitoids (Fig. 1c), which form three distinct age groups, spanning a c. 300 My interval: 1) Cadomian, 2) Early Ordovician and 3) Variscan.

(1) Granitoids of the first age group are represented by the $544 \pm 4$ Ma old Zawidów granodiorite (Białek et al. 2014), which belongs to the Cadomian Lusatian Massif, and by the Neoproterozoic (602-568 Ma: Oberc-Dziedzic et al. 2003; Klimas 2008; Klimas et al. 2009; Mazur et al. 2010) Strzelin and Nowolesie gneisses of the Strzelin Massif (Figs 1c; 2).

(2) The second age group includes metagranites (orthogneisses) of the Karkonosze-Izera Massif and the Orlica-Śnieżnik Dome, which are parts of the Sudetic Block, and by the Doboszowice gneisses and the pale Stachów gneisses which occur in the eastern part of the Fore-Sudetic Block. These granitoids were dated by various methods with ages spanning from Cambrian to Early Ordovician (515-480 Ma; e.g. Borkowska et al. 1980; Oliver et al. 1993; Turniak et al. 2000; Kröner et al. 2001; Oberc-Dziedzic et al. 2009, 2010b; Żelaźniewicz et al. 2009; Mazur et al. 2010).

(3) The third group comprises all Variscan granitoids. The oldest among them, c. 350-330 Ma in age, occurring in the Central Sudetes are connected with shear zones (Oberc-Dziedzic et al. 2015a and references therein). They are represented by hornblende-bearing granodiorites, tonalites, diorites of the Niemcza Zone, KłodzkoZłoty Stok Massif, and the Kudowa and Jawornik granites. Similar granitoids, known only from boreholes, occur within the Middle Odra Fault Zone (Dörr et al. 2006; Oberc-Dziedzic et al. 1999, 2015a).

In the West Sudetes, granitoids form two large bodies, namely, the Karkonosze and the Strzegom-Sobótka plutons, both younger than granitoids from the Central Sudetes. The two main rock-types of the Karkonosze Pluton were precisely dated by the ID-TIMS U-Pb zircon method at $312.5 \pm 0.3$ and $312.2 \pm 0.3 \mathrm{Ma}$ (Kryza et al.

Fig. 1a - Location of the Sudetes (black dot) in the European Variscides; b - Sketch map of the Bohemian Massif (ST - Saxothuringian, TB - Teplá-Barrandian, MO - Moldanubian zones, after Franke 2012) and Brunovistulicum (BV); MNST - Moldanubian-Nýznerov-Strzelin Thrust; quadrangle shows the location of the Sudetes; grey rectangle the position of the Strzelin Massif; c - Sketch map of the Sudetes; FSB - Fore-Sudetic Block, S - Sudetes, B - Bardo Structural Unit, NM - Niedźwiedź Massif; granitoids of the Middle Odra Fault Zone (known only from boreholes): GG - Gubin, SrG - Środa Śląska, SzG - Szprotawa, WrG - Wrocław; granitoids of the Fore-Sudetic Block: NG - Niemcza, SSG - Strzegom-Sobótka, StG - Strzelin, ZG - Žulova; granitoids of the Sudetes: KdG - Kudowa-Olešnice, KG - Karkonosze, KZG - Kłodzko-Złoty Stok; faults: UEFZ - Upper Elbe Fault Zone, MIF - Main Intra-Sudetic Fault, SMF - Sudetic Marginal Fault, SOF - Southern Odra Fault, NOF - Northern Odra Fault; thrusts: NT Nýznerov Thrust, ST - Strzelin Thrust. 


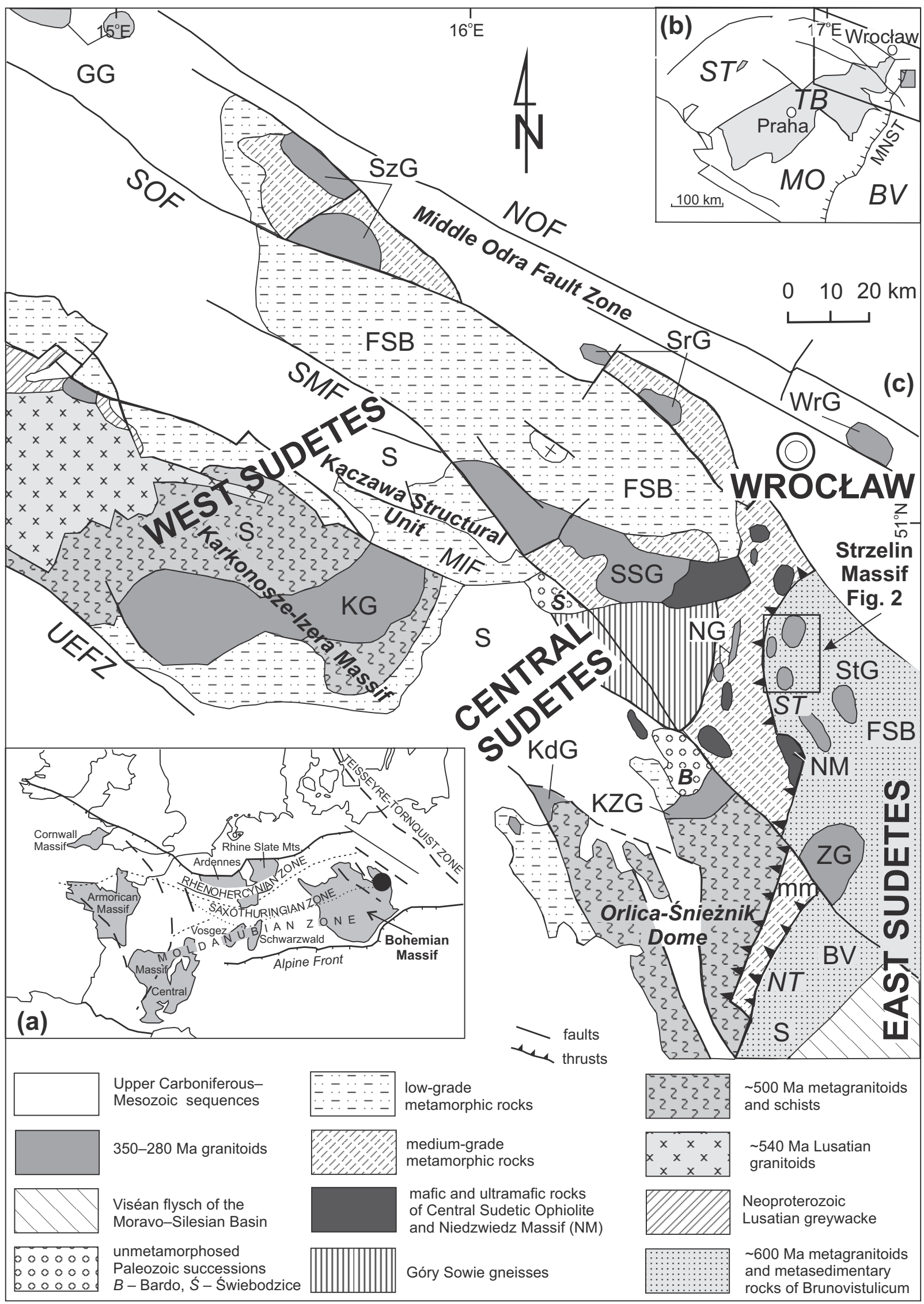


2014a), while the previous SIMS (SHRIMP) zircon data scattered mainly between 322 and 302 Ma (Awdankiewicz et al. 2010; Kryza et al. 2012, 2014b; Kusiak et al. 2009, 2014; Machowiak and Armstrong 2007), and the data obtained by the LA-ICP-MS method were 320-317 Ma (Žák et al. 2013). The ages of the Strzegom-Sobótka granitoids are 305-295 Ma based on SHRIMP U-Pb zircon data (Turniak et al. 2014).

Granitoids of the East Sudetes occur in the ForeSudetic Block. They form small intrusions of granites, tonalites and quartz diorites known from the Strzelin Massif and outcrops south of it, as well as from boreholes situated east of this massif. The granitoids were dated by various techniques between $324 \pm 4$ and $283 \pm 8$ Ma (Turniak et al. 2006; Pietranik and Waight 2008; Oberc-Dziedzic and Kryza 2012; Oberc-Dziedzic et al. 2010a, 2013a, 2015b). The relatively large Žulová Pluton, located south of the Strzelin Massif, belongs to the same generation (c. 292 Ma, LA-ICP-MS U-Pb zircon age; Laurent et al. 2014).

All three major age groups of granitoids occur in the westerly Karkonosze-Izera Massif, and the easterly Strzelin Massif (Fig. 1c). However, their mutual relationships differ in both parts of Sudetes. In the West Sudetes, the Cadomian Zawidów granodiorites were intruded by the 500 Ma old Rumburk granite (Białek et al. 2014 and references therein) and the $500 \mathrm{Ma}$ igneous protolith of the Izera orthogneisses. In turn, the Karkonosze granite intruded the Izera orthogneisses but not the Zawidów granodiorite. In the Strzelin Massif, the 500 Ma gneisses were thrust over the Neoproterozoic gneisses during Variscan tectonism. Subsequently, both the Neoproterozoic and Early Ordovician gneisses were intruded by the Variscan granitoids.

\subsection{The Strzelin Massif}

The Strzelin Massif (Fig. 2) comprises poorly exposed crystalline rocks in the eastern part of the Fore-Sudetic Block, about $40 \mathrm{~km}$ south of Wrocław (Fig. 1c).

The Strzelin Massif is composed of two tectonic units separated by the Strzelin Thrust considered as a northern prolongation of the Moldanubian overthrust (ObercDziedzic and Madej 2002; Oberc-Dziedzic et al. 2005, 2010a). The footwall of the Strzelin Thrust (the lower tectonic unit) is formed by the Strzelin Complex whereas the hanging wall (the upper tectonic unit) is composed of the Stachów Complex (Fig. 3). The Strzelin Complex belongs to the (para-autochtonous) Brunovistulicum (Oberc-Dziedzic et al. 2003, 2005), whereas the overlying Stachów Complex can be correlated with the Moldanubian zone sensu Aleksandrowski and Mazur (2002) (Oberc-Dziedzic and Madej 2002; Oberc-Dziedzic et al. $2005,2010 a)$, or possibly with the Saxothuringian Zone based on the presence of $c$. 500 Ma old orthogneisses that are commonly found in this zone (e.g. Oberc-Dziedzic et al. 2009, 2010b; Żelaźniewicz et al. 2009). The rocks of both complexes were metamorphosed and deformed during the Variscan Orogeny (Oberc 1966; Oberc-Dziedzic et al. 2010a) and intruded by late- and post-tectonic granitoids.

\section{Analytical techniques}

The detailed petrographic descriptions of gneisses and the Variscan granitoids occurring in the Strzelin Massif were published in the articles by the above-cited authors, based on several hundreds of thin sections.

Fifty five samples were analysed for major and trace elements using the combined ICP-OES and ICP-MS techniques (Actlabs, Vancouver, Canada, code "4Lithores": http://www.actlabs.com). Among 55 analyses, 32 results are new (Tabs 1-3) and 23 have been published previously (Electronic supplementary material, ESM 1-4).

The published $\mathrm{Rb}-\mathrm{Sr}$ results for the Strzelin granitoids (Oberc-Dziedzic et al. 1996) are presented as ESM 5.

Twenty-four samples have been analysed by C. Pin for $\mathrm{Sm}-\mathrm{Nd}$ isotopes, following procedures described in detail by Pin and Santos Zalduegui (1997). Briefly, the samples were spiked with a mixed ${ }^{149} \mathrm{Sm}-{ }^{150} \mathrm{Nd}$ enriched isotopic tracer for the isotope dilution method and dissolved. The complete decomposition of REE-rich refractory accessory minerals was ensured either by treating any solid residue left after HF dissolution in a high-pressure Teflon bomb, or by sample fusion with a $\mathrm{LiBO}_{2}$ flux. Then, $\mathrm{Nd}$ and $\mathrm{Sm}$ were separated from matrix elements and from each other by using a combination of cation-exchange chromatography and extraction chromatography. Samarium was loaded on a Ta filament and the ${ }^{149} \mathrm{Sm} /{ }^{147} \mathrm{Sm}$ ratio measured on the $\mathrm{Sm}^{+}$ion beam by single-collector VG54e thermal-ionization mass spectrometer (TIMS). Neodymium was loaded on a triple Ta-Re-Ta filament assembly and measured as $\mathrm{Nd}^{+}$by using an upgraded VG54e TIMS (Clermont-Ferrand) operated in dynamic triplecollection mode, providing ${ }^{143} \mathrm{Nd} /{ }^{144} \mathrm{Nd}$ (and ${ }^{150} \mathrm{Nd} /{ }^{144} \mathrm{Nd}$ for isotope dilution determination of $\mathrm{Nd}$ concentration) ratios. Alternatively, $\mathrm{Nd}$ was loaded on a double Re filament assembly and measured with a TRITON TIMS (GIS Lab at Nîmes University) in static multi-collection mode. Mass fractionation was corrected by normalization to ${ }^{146} \mathrm{Nd} /{ }^{144} \mathrm{Nd}=0.7219$, taking into account the spike contribution. Repeat measurements of the La Jolla and JNdi-1 isotopic standards (Tanaka et al. 2000) were used to normalize the data to a ${ }^{143} \mathrm{Nd} /{ }^{144} \mathrm{Nd}$ value of 0.512110 . The initial ${ }^{143} \mathrm{Nd} /{ }^{144} \mathrm{Nd}$ ratios are expressed as $\varepsilon \mathrm{Nd}_{\mathrm{i}}$ values, corrected for in situ decay of ${ }^{147} \mathrm{Sm}$ assuming ages established by the U-Pb SHRIMP method for each particular 
Three generations of granitoids in the Strzelin Massif, implications for crustal evolution

group of rocks, and using the CHUR parameters of Jacobsen and Wasserburg (1980). The model ages were calculated relative to the Depleted Mantle model of De Paolo (1981a, b).

Among twenty four Sm-Nd isotope results, 13 analyses of gneisses are new (Tab. 4), while the rest have been published already (ESM 6; Oberc-Dziedzic et al. 2010a, 2013a, 2015b).

\section{Petrography of the orthogneisses and granites of the Strzelin Massif}

\subsection{The gneisses of the Strzelin Complex}

The Strzelin orthogneisses (Fig. 2), with ${ }^{206} \mathrm{~Pb} /{ }^{238} \mathrm{U}$ SHRIMP zircon ages of $600 \pm 7$ and $568 \pm 7 \mathrm{Ma}$ (Oberc-Dziedzic et al. 2003) are light grey, fineand medium-grained rocks, with random, streaky, banded or pencil structure. Their characteristic feature is the presence of $\mathrm{K}$-feldspar and plagioclase grains up to $1 \mathrm{~cm}$ in size. The matrix is composed of sericitized plagioclase, small grains of $\mathrm{K}$-feldspar, lenticular aggregates of quartz grains and flakes of biotite and muscovite. The Strzelin gneisses contain amphibolite layers, up to several meters thick, parallel to the foliation of the gneisses. They probably represent Early Paleozoic mafic dykes of within-plate geochemical characteristics (Szczepański and Oberc-Dziedzic 1998), cross-cutting the granitic protolith of the gneisses.

The Nowolesie gneisses (Fig. 2) with ${ }^{206} \mathrm{~Pb} /{ }^{238} \mathrm{U}$ SHRIMP zircon ages of $602 \pm 7 \mathrm{Ma}$, $587 \pm 4$ Ma (Klimas 2008; Mazur et al. 2010) are light grey, fine-grained, streaky or banded
Tab. 1 Chemical analyses of the Strzelin Complex orthogneisses in the Strzelin Massif

\begin{tabular}{|c|c|c|c|c|c|c|c|c|c|c|}
\hline \multirow[b]{2}{*}{ wt. $\%$} & \multicolumn{6}{|c|}{ Strzelin orthogneisses } & \multicolumn{4}{|c|}{ Nowolesie sillimanite gneisses } \\
\hline & B1 26.5 & $210-50$ & S 80 & S 34 & WL 2.11 & WL 3.15 & SK 1 & N 2 & N 24 & $\mathrm{~N} 4.3$ \\
\hline $\mathrm{SiO}_{2}$ & 72.66 & 70.99 & 75.32 & 74.58 & 74.18 & 71.85 & 70.26 & 75.36 & 74.53 & 67.42 \\
\hline $\mathrm{TiO}_{2}$ & 0.15 & 0.16 & 0.08 & 0.11 & 0.10 & 0.13 & 0.18 & 0.04 & 0.10 & 0.39 \\
\hline $\mathrm{Al}_{2} \mathrm{O}_{3}$ & 15.54 & 15.69 & 14.28 & 14.99 & 14.70 & 14.50 & 16.17 & 14.41 & 14.24 & 16.74 \\
\hline $\mathrm{Fe}_{2} \mathrm{O}_{3}$ & 1.66 & 1.28 & 0.69 & 0.81 & 1.95 & 4.25 & 1.54 & 1.44 & 0.67 & 4.35 \\
\hline $\mathrm{MnO}$ & 0.03 & 0.02 & 0.02 & 0.01 & 0.02 & 0.04 & 0.03 & 0.05 & 0.01 & 0.10 \\
\hline $\mathrm{MgO}$ & 0.51 & 0.48 & 0.15 & 0.38 & 0.44 & 0.60 & 0.94 & 0.21 & 0.25 & 1.52 \\
\hline $\mathrm{CaO}$ & 1.25 & 1.90 & 0.90 & 0.35 & 0.44 & 0.49 & 1.08 & 0.99 & 0.29 & 3.32 \\
\hline $\mathrm{Na}_{2} \mathrm{O}$ & 4.64 & 4.75 & 3.56 & 5.91 & 3.73 & 4.57 & 5.00 & 3.95 & 4.40 & 4.17 \\
\hline $\mathrm{K}_{2} \mathrm{O}$ & 2.98 & 3.15 & 4.27 & 2.03 & 4.58 & 3.99 & 3.80 & 3.77 & 4.89 & 2.05 \\
\hline $\mathrm{P}_{2} \mathrm{O}_{5}$ & 0.05 & 0.04 & 0.03 & 0.05 & 0.07 & 0.10 & 0.09 & 0.06 & 0.03 & 0.18 \\
\hline LOI & 0.40 & 1.50 & 0.70 & 0.80 & 0.31 & 0.00 & 0.80 & 0.12 & 0.60 & 0.19 \\
\hline Total & 99.87 & 99.96 & 100.00 & 100.02 & 100.52 & 100.52 & 99.89 & 100.40 & 100.01 & 100.43 \\
\hline $\mathrm{A} / \mathrm{CNK}$ & 1.18 & 1.10 & 1.18 & 1.19 & 1.23 & 1.14 & 1.13 & 1.16 & 1.09 & 1.11 \\
\hline $\mathrm{A} / \mathrm{NK}$ & 1.43 & 1.40 & 1.36 & 1.26 & 1.32 & 1.22 & 1.31 & 1.36 & 1.14 & 1.84 \\
\hline \multicolumn{11}{|l|}{$\mathrm{ppm}$} \\
\hline $\mathrm{Ba}$ & 925 & 860 & 1097 & 572 & 953 & 915 & 585 & 1083 & 1337 & 913 \\
\hline $\mathrm{Rb}$ & 108 & 79 & 115 & 46 & 133 & 86 & 195 & 122 & 103 & 142 \\
\hline $\mathrm{Sr}$ & 366 & 479 & 249 & 130 & 168 & 220 & 283 & 218 & 214 & 285 \\
\hline $\mathrm{Y}$ & 6.9 & 4.6 & 7.0 & 9.6 & 4.0 & 8.9 & 7.2 & 13.0 & 3.2 & 16.0 \\
\hline $\mathrm{Zr}$ & 91.0 & 100.9 & 61.3 & 58.6 & 65.0 & 73.0 & 103.1 & 36.0 & 53.0 & 91.0 \\
\hline $\mathrm{Nb}$ & 5.7 & 6.7 & 5.1 & 6.3 & 5.5 & 5.3 & 7.6 & 6.1 & 4.5 & 13.0 \\
\hline $\mathrm{Th}$ & 4.76 & 3.10 & 6.10 & 6.90 & 4.22 & 4.75 & 4.50 & 4.06 & 3.40 & 5.06 \\
\hline $\mathrm{Pb}$ & & 2.2 & 2.4 & 3.8 & 17.0 & 9.0 & 1.7 & 12.0 & 3.3 & 5.0 \\
\hline $\mathrm{Ga}$ & 18.0 & 18.9 & 14.9 & 16.4 & 18.0 & 15.0 & 19.0 & 18.0 & 14.3 & 20.0 \\
\hline $\mathrm{Zn}$ & & 25 & 17 & 19 & 92 & 36 & 41 & & 14 & 84 \\
\hline $\mathrm{Cu}$ & & 2.1 & 1.0 & 5.8 & 22.0 & 37.0 & 3.1 & 13.0 & 2.8 & 12.0 \\
\hline $\mathrm{V}$ & 8 & $<5$ & $<5$ & 5 & & 6 & 9 & & $<5$ & 53 \\
\hline $\mathrm{Cr}$ & & 10 & 10 & 10 & & & 10 & & 10 & \\
\hline $\mathrm{Hf}$ & 2.5 & 2.9 & 1.9 & 2.2 & 2.3 & 2.1 & 3.3 & 1.5 & 1.7 & 3.1 \\
\hline $\mathrm{Cs}$ & 1.6 & 0.7 & 0.6 & 0.3 & 0.7 & 0.4 & 8.7 & 2.4 & 1.4 & 4.3 \\
\hline $\mathrm{Sc}$ & 2 & 2 & 2 & 2 & 2 & 2 & 3 & 2 & 2 & 7 \\
\hline $\mathrm{Ta}$ & 0.38 & 0.40 & 0.40 & 0.70 & 0.50 & 0.36 & 0.70 & 0.50 & 0.30 & 1.10 \\
\hline Co & & 84.5 & 118.1 & 82.4 & 3.0 & 4.0 & 114.4 & 2.0 & 96.8 & 5.0 \\
\hline $\mathrm{Be}$ & 2 & 1 & 2 & 2 & & & 3 & 2 & 1 & 3 \\
\hline $\mathrm{U}$ & 0.78 & 0.70 & 0.60 & 0.80 & 0.57 & & 1.60 & 1.13 & 0.50 & 2.06 \\
\hline $\mathrm{Sn}$ & & $<1$ & 1 & $<1$ & 5 & & 4 & 3 & 1 & 2 \\
\hline Mo & & 0.1 & $<0.1$ & 0.3 & 0.0 & & 0.1 & 0.0 & 0.1 & 0.0 \\
\hline $\mathrm{La}$ & 18.5 & 20.8 & 19.1 & 19.1 & 10.8 & 16.6 & 18.4 & 11.0 & 19.0 & 17.8 \\
\hline $\mathrm{Ce}$ & 33.5 & 40.3 & 35.4 & 40.0 & 20.0 & 32.9 & 37.2 & 22.0 & 37.6 & 35.0 \\
\hline $\operatorname{Pr}$ & 3.46 & 3.77 & 3.31 & 3.63 & 2.13 & 3.28 & 3.79 & 2.47 & 3.36 & 3.71 \\
\hline $\mathrm{Nd}$ & 12.3 & 12.6 & 11.8 & 13.8 & 7.2 & 11.8 & 14.8 & 9.0 & 11.0 & 14.0 \\
\hline $\mathrm{Sm}$ & 2.04 & 2.00 & 1.70 & 2.60 & 1.32 & 2.04 & 2.40 & 1.92 & 1.90 & 2.93 \\
\hline $\mathrm{Eu}$ & 0.598 & 0.700 & 0.470 & 0.470 & 0.364 & 0.444 & 0.560 & 0.335 & 0.780 & 0.816 \\
\hline $\mathrm{Gd}$ & 1.35 & 1.38 & 1.25 & 1.98 & 1.17 & 1.82 & 1.68 & 1.99 & 1.01 & 2.80 \\
\hline $\mathrm{Tb}$ & 0.21 & 0.17 & 0.21 & 0.35 & 0.14 & 0.26 & 0.27 & 0.32 & 0.16 & 0.46 \\
\hline Dy & 1.19 & 0.87 & 1.19 & 1.92 & 0.70 & 1.58 & 1.35 & 1.91 & 0.92 & 2.68 \\
\hline Ho & 0.22 & 0.17 & 0.19 & 0.35 & 0.13 & 0.32 & 0.26 & 0.41 & 0.11 & 0.56 \\
\hline $\mathrm{Er}$ & 0.66 & 0.51 & 0.69 & 0.92 & 0.37 & 0.90 & 0.71 & 1.30 & 0.27 & 1.78 \\
\hline $\mathrm{Tm}$ & 0.09 & 0.08 & 0.11 & 0.14 & 0.05 & 0.12 & 0.12 & 0.21 & 0.06 & 0.29 \\
\hline $\mathrm{Yb}$ & 0.68 & 0.46 & 0.73 & 0.71 & 0.34 & 0.82 & 0.82 & 1.41 & 0.25 & 1.83 \\
\hline $\mathrm{Lu}$ & 0.100 & 0.050 & 0.120 & 0.120 & 0.056 & 0.116 & 0.120 & 0.205 & 0.050 & 0.298 \\
\hline$\Sigma \mathrm{REE}$ & 74.9 & 83.9 & 76.3 & 86.1 & 44.7 & 73.0 & 82.5 & 54.5 & 76.5 & 85.0 \\
\hline $\mathrm{La}_{\mathrm{N}} / \mathrm{Yb}_{\mathrm{N}}$ & 19.5 & 32.4 & 18.8 & 19.3 & 22.8 & 14.5 & 16.1 & 5.6 & 54.5 & 7.0 \\
\hline $\mathrm{Eu} / \mathrm{Eu}^{*}$ & 1.10 & 1.29 & 0.99 & 0.63 & 0.90 & 0.70 & 0.85 & 0.52 & 1.72 & 0.87 \\
\hline
\end{tabular}

B1 26.5 (N50³9'43.9", E176'12.5"); 210-50 (Gęsiniec q. N5045'19.5", E17²4'); S 80 (N5043'36.3", E17ํ'1.4"); S 34 (N5044'52.3", E177'29.1"); WL 2.11 (N5040'46.8", E1659'29.6"); WL 3.15

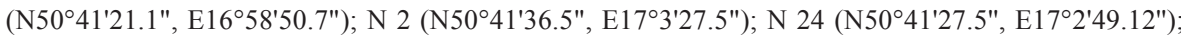
$\mathrm{N} 4.3\left(\mathrm{~N} 50^{\circ} 41^{\prime} 42.9^{\prime \prime}, \mathrm{E} 17^{\circ} 4^{\prime} 14.3^{\prime \prime}\right)$ 
Tab. 2 Chemical analyses of the Stachów Complex orthogneisses in the Strzelin Massif

\begin{tabular}{|c|c|c|c|c|c|c|c|c|c|c|c|c|c|c|c|c|}
\hline \multirow[b]{4}{*}{ wt. $\%$} & \multicolumn{3}{|c|}{ The Strzelin Hills } & \multicolumn{13}{|c|}{ The Lipowe Hills } \\
\hline & \multirow{3}{*}{ S 21} & \multirow{3}{*}{$310 \mathrm{C}$} & \multirow{3}{*}{$300 \mathrm{~V} 1$} & \multirow{3}{*}{$\begin{array}{c}\text { WL } \\
7.11\end{array}$} & \multirow{3}{*}{$\begin{array}{c}\text { WL } \\
5.612\end{array}$} & \multirow{3}{*}{$\begin{array}{l}\text { ST } 2 \\
27.8\end{array}$} & \multirow{3}{*}{$\begin{array}{c}\text { ST } 2 \\
83\end{array}$} & \multirow{3}{*}{$\begin{array}{c}\text { ST } 1 \\
41\end{array}$} & \multirow{3}{*}{$\begin{array}{c}\text { ST } 2 \\
79\end{array}$} & \multicolumn{3}{|c|}{ with garnet } & \multicolumn{2}{|c|}{ aplitic } & Henrykó & $\mathrm{W}$ gneiss \\
\hline & & & & & & & & & & WL & ST 1 & ST 2 & WL & WL & $\mathrm{HR}$ & HR \\
\hline & & & & & & & & & & 6.11 & 86 & 233 & 9.11 & 11.12 & 1.14 & $302-4$ \\
\hline $\mathrm{SiO}_{2}$ & 69.24 & 77.07 & 76.08 & 71.92 & 69.50 & 72.10 & 68.43 & 71.71 & 69.81 & 76.42 & 76.40 & 75.56 & 74.32 & 77.28 & 70.15 & 64.41 \\
\hline $\mathrm{TiO}_{2}$ & 0.50 & 0.16 & 0.17 & 0.42 & 0.43 & 0.39 & 0.43 & 0.43 & 0.52 & 0.13 & 0.08 & 0.09 & 0.08 & 0.08 & 0.46 & 0.69 \\
\hline $\mathrm{Al}_{2} \mathrm{O}_{3}$ & 14.40 & 12.26 & 12.23 & 14.58 & 14.85 & 14.03 & 14.76 & 15.05 & 15.25 & 12.49 & 12.66 & 13.14 & 13.35 & 12.95 & 14.98 & 15.54 \\
\hline $\mathrm{Fe}_{2} \mathrm{O}_{3}$ & 4.83 & 1.35 & 1.21 & 2.50 & 2.59 & 2.59 & 3.27 & 3.14 & 3.68 & 2.40 & 1.33 & 1.42 & 4.60 & 1.50 & 3.99 & 5.96 \\
\hline $\mathrm{MnO}$ & 0.06 & 0.01 & 0.01 & 0.02 & 0.03 & 0.03 & 0.06 & 0.04 & 0.04 & 0.05 & 0.04 & 0.04 & 0.04 & 0.02 & 0.03 & 0.06 \\
\hline $\mathrm{MgO}$ & 1.10 & 0.12 & 0.15 & 0.36 & 0.79 & 0.73 & 0.72 & 0.90 & 1.05 & 0.24 & 0.15 & 0.17 & 0.65 & 0.76 & 1.43 & 3.10 \\
\hline $\mathrm{CaO}$ & 1.46 & 0.57 & 0.55 & 0.64 & 1.78 & 0.97 & 0.93 & 2.01 & 1.85 & 0.90 & 0.44 & 0.41 & 0.45 & 0.24 & 0.62 & 0.33 \\
\hline $\mathrm{Na}_{2} \mathrm{O}$ & 3.11 & 2.92 & 2.35 & 2.57 & 3.46 & 2.87 & 3.64 & 3.69 & 3.58 & 3.22 & 2.63 & 3.55 & 1.79 & 1.59 & 3.90 & 3.60 \\
\hline $\mathrm{K}_{2} \mathrm{O}$ & 4.82 & 5.52 & 6.32 & 4.92 & 3.99 & 5.46 & 3.94 & 2.62 & 4.03 & 3.98 & 5.69 & 5.24 & 4.33 & 5.16 & 2.86 & 1.73 \\
\hline $\mathrm{P}_{2} \mathrm{O}_{5}$ & 0.19 & 0.05 & 0.06 & 0.13 & 0.10 & 0.10 & 0.10 & 0.16 & 0.16 & 0.12 & 0.13 & 0.15 & 0.02 & 0.03 & 0.15 & 0.16 \\
\hline LOI & 0.08 & 0.10 & 0.70 & 0.70 & 0.05 & 0.36 & 3.08 & 0.22 & 0.28 & 0.11 & 0.25 & 0.11 & 0.00 & 0.74 & 1.60 & 4.40 \\
\hline Total & 99.79 & 100.13 & 99.83 & 98.76 & 97.57 & 99.63 & 99.36 & 99.97 & 100.251 & 100.06 & 99.80 & 99.88 & 99.63 & 100.34 & 100.17 & 99.98 \\
\hline$\overline{\mathrm{A} / \mathrm{CNK}}$ & 1.10 & 1.04 & 1.00 & 1.36 & 1.12 & 1.13 & 1.23 & 1.20 & 1.12 & 1.11 & 1.12 & 1.07 & 1.58 & 1.50 & 1.43 & 1.80 \\
\hline $\mathrm{A} / \mathrm{NK}$ & 1.39 & 1.14 & 1.10 & 1.52 & 1.48 & 1.32 & 1.44 & 1.69 & 1.49 & 1.30 & 1.21 & 1.14 & 1.75 & 1.58 & 1.57 & 2.00 \\
\hline ppm & & & & & & & & & & & & & & & & \\
\hline $\mathrm{Ba}$ & 784 & 261 & 387 & 936 & 1310 & 781 & 843 & 780 & 1150 & 397 & 170 & 150 & 142 & 126 & 666 & 389 \\
\hline $\mathrm{Rb}$ & 152 & 154 & 145 & 150 & 114 & 185 & 122 & 88 & 130 & 105 & 172 & 181 & 227 & 207 & 60 & 55 \\
\hline $\mathrm{Sr}$ & 103 & 31 & 50 & 108 & 161 & 127 & 131 & 165 & 142 & 154 & 33 & 48 & 21 & 37 & 84 & 64 \\
\hline $\mathrm{Y}$ & 34.0 & 48.7 & 37.1 & 42.7 & 35.8 & 31.3 & 43.3 & 46.3 & 43.9 & 44.0 & 23.1 & 22.8 & 80.5 & 54.2 & 41.2 & 25.7 \\
\hline $\mathrm{Zr}$ & 173.0 & 129.2 & 130.8 & 198.0 & 205.0 & 199.0 & 205.0 & 201.0 & 231.0 & 80.0 & 69.0 & 79.0 & 96.0 & 90.0 & 225.0 & 188.2 \\
\hline $\mathrm{Nb}$ & 11.0 & 6.4 & 4.8 & 11.2 & 9.3 & 10.2 & 8.0 & 8.4 & 9.7 & 3.7 & 2.4 & 4.0 & 11.7 & 8.4 & 11.4 & 12.1 \\
\hline Th & 13.9 & 14.8 & 17.1 & 17.6 & 17.6 & 21.5 & 18.3 & 17.2 & 17.8 & 6.5 & 5.1 & 3.3 & 27.6 & 23.6 & 17.7 & 10.7 \\
\hline $\mathrm{Ga}$ & 17.0 & 16.9 & 16.0 & 17.0 & 18.4 & 18.0 & 19.0 & 18.0 & 19.0 & 14.0 & 15.0 & 16.0 & 26.0 & 23.0 & 20.0 & 19.6 \\
\hline $\mathrm{Zn}$ & 60 & 17 & 16 & & 49 & & & & & & & & & & & 54 \\
\hline V & 53 & 5 & 7 & 32 & 37 & 33 & 38 & 36 & 47 & 9 & 8 & 7 & & & 44 & 86 \\
\hline $\mathrm{Cr}$ & 48 & 10 & 10 & 22 & 10 & & & & & & & & & & 21 & 12 \\
\hline $\mathrm{Hf}$ & 6.3 & 4.7 & 4.6 & 6.3 & 5.8 & 5.8 & 5.8 & 5.9 & 6.8 & 3.2 & 2.3 & 2.6 & 4.8 & 4.2 & 6.7 & 5.1 \\
\hline Cs & 3.7 & 2.6 & 1.4 & 3.5 & 3.1 & 2.6 & 2.5 & 3.5 & 3.0 & 2.0 & 1.4 & 2.4 & 6.3 & 1.0 & 0.5 & 0.8 \\
\hline $\mathrm{Sc}$ & 9 & 4 & 3 & 8 & & 6 & 8 & 8 & 9 & 5 & 5 & 4 & 5 & 4 & 9 & 13 \\
\hline $\mathrm{Ta}$ & 0.90 & 0.40 & 0.30 & 0.58 & 0.70 & 1.03 & 0.60 & 0.59 & 0.74 & 0.50 & 0.21 & 0.72 & 1.58 & 1.31 & 0.87 & 0.90 \\
\hline Co & 296.0 & 122.9 & 88.4 & 5.0 & 51.2 & & & & & 3.0 & & & 5.0 & & 7.0 & 48.4 \\
\hline $\mathrm{U}$ & 3.59 & 3.90 & 4.00 & & 2.67 & 6.98 & 2.72 & 10.10 & 3.06 & 2.01 & 2.47 & 6.57 & & 4.38 & & 1.80 \\
\hline $\mathrm{La}$ & 30.4 & 26.3 & 25.9 & 39.7 & 40.0 & 35.0 & 39.9 & 40.4 & 39.9 & 14.2 & 8.4 & 7.9 & 38.4 & 35.5 & 37.9 & 40.0 \\
\hline $\mathrm{Ce}$ & 65.0 & 64.7 & 59.6 & 84.6 & 78.6 & 72.0 & 79.9 & 80.4 & 80.2 & 28.0 & 17.9 & 17.0 & 74.6 & 75.5 & 80.8 & 92.6 \\
\hline $\operatorname{Pr}$ & 7.61 & 6.61 & 7.11 & 8.87 & 9.76 & 8.03 & 8.94 & 9.03 & 8.93 & 3.05 & 2.02 & 1.82 & 10.30 & 9.52 & 8.53 & 9.08 \\
\hline $\mathrm{Nd}$ & 28.7 & 24.7 & 27.7 & 35.0 & 35.0 & 30.3 & 34.1 & 34.7 & 34.8 & 11.7 & 7.6 & 6.7 & 39.4 & 36.2 & 33.8 & 35.3 \\
\hline $\mathrm{Sm}$ & 6.24 & 6.10 & 5.90 & 7.42 & 7.57 & 6.28 & 6.80 & 7.24 & 7.34 & 2.74 & 1.96 & 1.58 & 9.60 & 8.99 & 7.03 & 6.70 \\
\hline $\mathrm{Eu}$ & 0.832 & 0.300 & 0.250 & 0.995 & 1.130 & 0.650 & 0.905 & 1.010 & 1.020 & 0.213 & 0.150 & 0.137 & 0.088 & 0.079 & 0.999 & 1.320 \\
\hline Gd & 6.51 & 6.42 & 5.62 & 7.61 & 7.55 & 5.35 & 6.06 & 6.78 & 6.58 & 3.33 & 1.92 & 1.52 & 10.80 & 7.98 & 7.26 & 5.38 \\
\hline $\mathrm{Tb}$ & 1.06 & 1.26 & 1.09 & 1.36 & 1.21 & 0.93 & 1.09 & 1.28 & 1.22 & 0.87 & 0.52 & 0.44 & 2.30 & 1.60 & 1.25 & 0.83 \\
\hline Dy & 5.91 & 7.87 & 5.95 & 8.30 & 6.66 & 5.44 & 6.87 & 7.81 & 7.38 & 6.67 & 3.67 & 3.53 & 15.00 & 10.10 & 7.95 & 4.80 \\
\hline Ho & 1.22 & 1.63 & 1.25 & 1.69 & 1.33 & 0.99 & 1.40 & 1.53 & 1.43 & 1.59 & 0.74 & 0.74 & 3.15 & 1.95 & 1.62 & 0.92 \\
\hline Er & 3.56 & 4.92 & 3.53 & 4.70 & 3.62 & 2.94 & 4.68 & 4.55 & 4.44 & 5.29 & 2.42 & 2.40 & 9.23 & 6.12 & 4.63 & 2.64 \\
\hline $\mathrm{Tm}$ & 0.53 & 0.68 & 0.53 & 0.69 & 0.48 & 0.43 & 0.69 & 0.69 & 0.62 & 0.94 & 0.45 & 0.38 & 1.46 & 0.87 & 0.67 & 0.40 \\
\hline $\mathrm{Yb}$ & 3.11 & 4.39 & 3.05 & 4.16 & 3.03 & 2.66 & 4.47 & 3.97 & 3.94 & 5.75 & 2.67 & 2.68 & 8.97 & 5.89 & 4.23 & 2.35 \\
\hline $\mathrm{Lu}$ & 0.440 & 0.630 & 0.460 & 0.578 & 0.420 & 0.382 & 0.660 & 0.553 & 0.548 & 0.796 & 0.378 & 0.370 & 1.200 & 0.830 & 0.575 & 0.370 \\
\hline$\overline{\Sigma R E E}$ & 161.1 & 156.5 & 147.9 & 205.7 & 196.4 & 171.4 & 196.5 & 199.9 & 198.4 & 85.1 & 50.8 & 47.1 & 224.5 & 201.1 & 197.3 & 202.7 \\
\hline $\mathrm{La}_{\mathrm{N}} / \mathrm{Yb}_{\mathrm{N}}$ & 7.0 & 4.3 & 6.1 & 6.9 & 9.5 & 9.4 & 6.4 & 7.3 & 7.3 & 1.8 & 2.3 & 2.1 & 3.1 & 4.3 & 6.4 & 12.2 \\
\hline $\mathrm{Eu} / \mathrm{Eu}^{*}$ & 0.40 & 0.15 & 0.13 & 0.40 & 0.46 & 0.34 & 0.43 & 0.44 & 0.45 & 0.22 & 0.24 & 0.27 & 0.03 & 0.03 & 0.43 & 0.67 \\
\hline
\end{tabular}

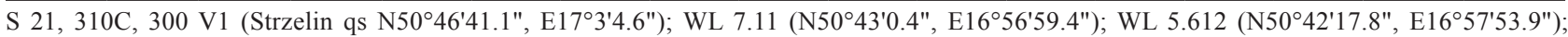
ST 2 27.8, ST 2 83, ST 2 79, ST2 233 (N5043'1.4", E1657'58.6"); ST 1 41, ST 186 (N5043'33.8", E1657'52.2"); WL 6.11 (N5043'23.4", E16 57'51.8"); WL 9.11 (N5043'37.8", E1657'9.6"); WL 11.12 (N5043'45.2", E1657'19.1"); HR 1.14, HR 302-4 (N50³8'41.9", E16 59'51.2") 


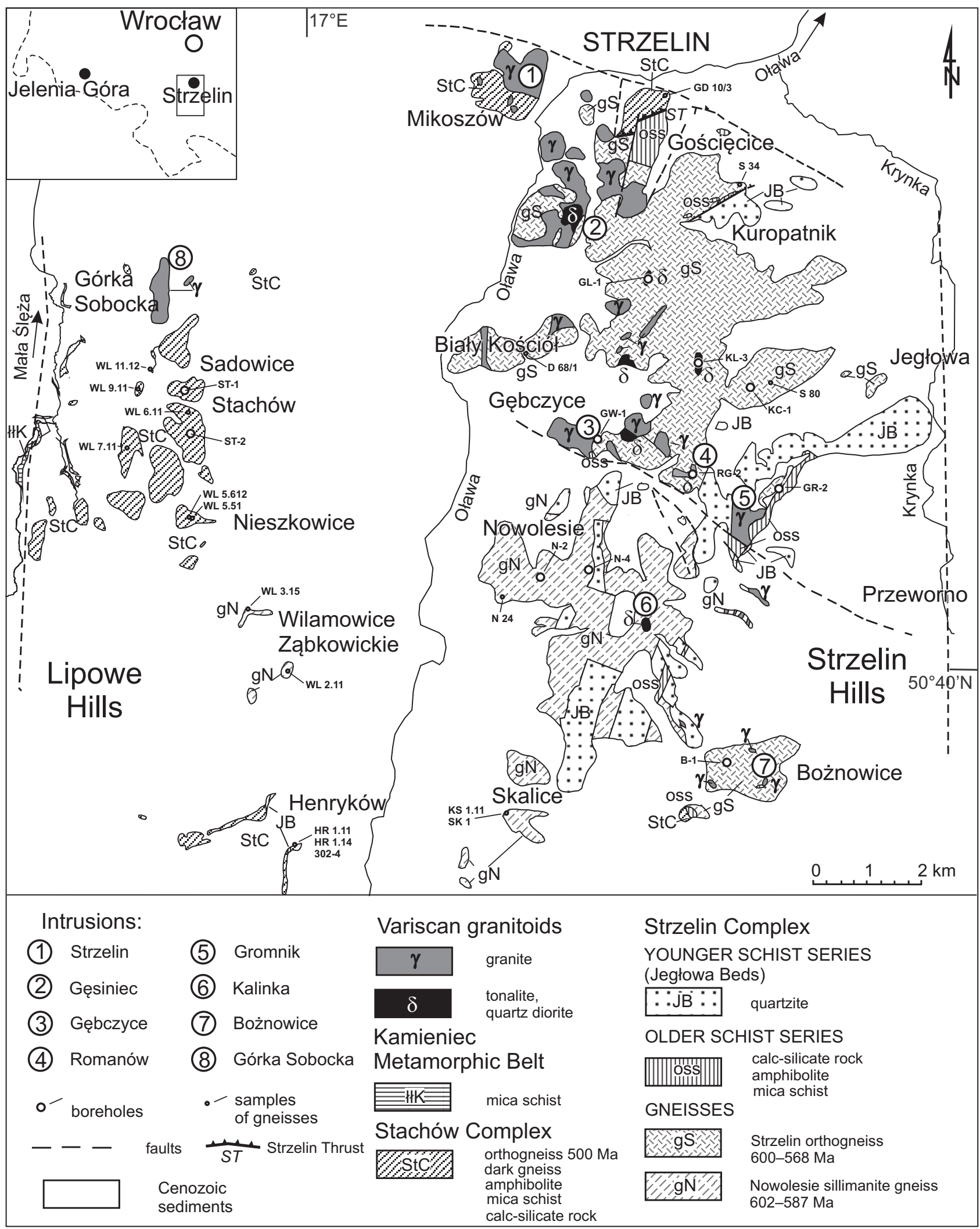

Fig. 2 Geological map of the Strzelin Massif (compiled by Oberc-Dziedzic and Madej 2002; based on Wójcik 1968; Wroński 1973; Badura 1979; Oberc et al. 1988). Inset: Lower Silesia; rectangle shows the position of the Strzelin Massif. The samples indicated refer to new analyses (Tabs $1-4)$ as well as the pre-existing ones given in the Electronic Appendix. GPS coordinates of the analysed samples are listed in Tabs 1-4 and in the Electronic supplementary materials (ESM). 


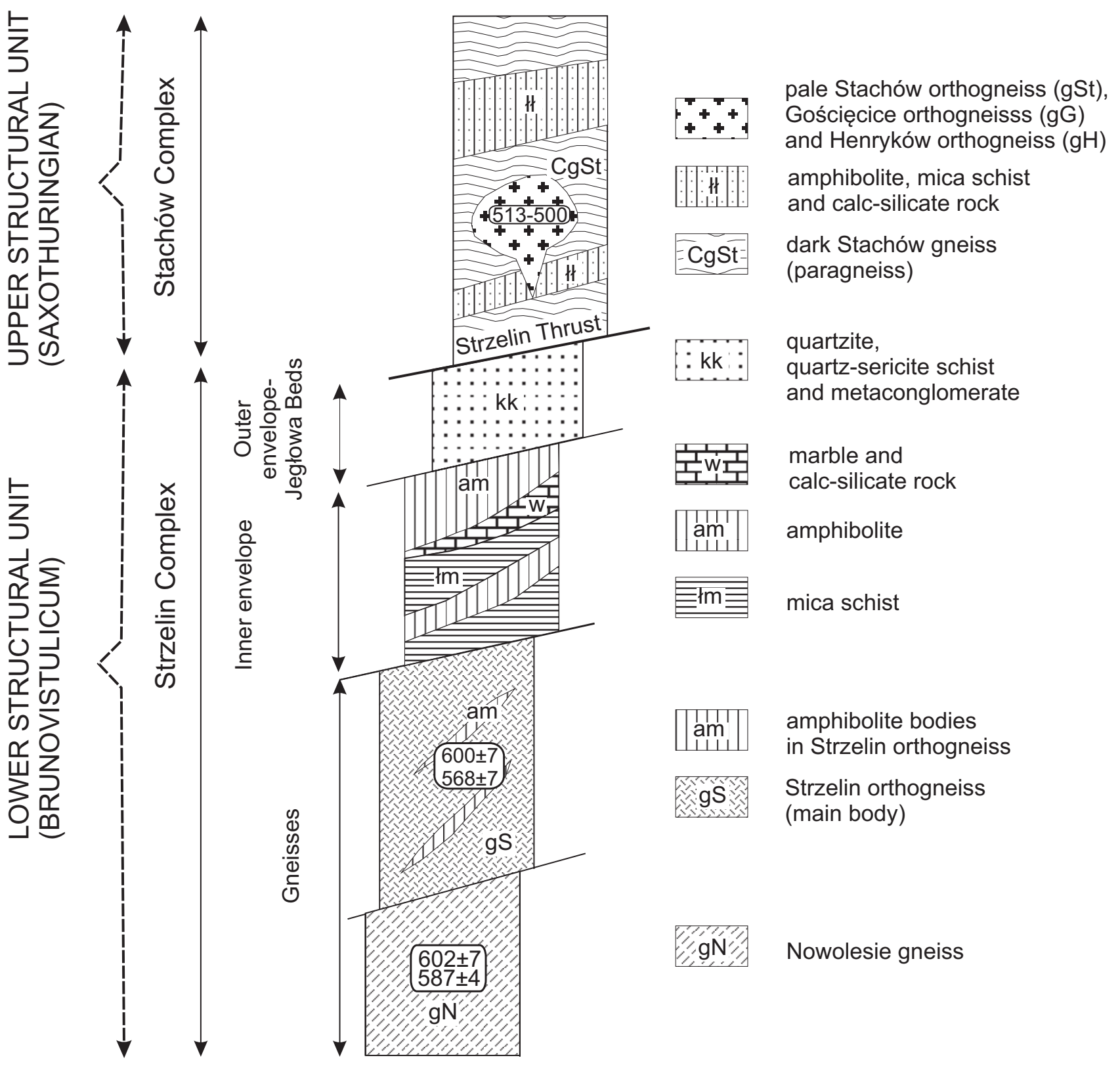

Fig. 3 Simplified tectonostratigraphic division of rock complexes composing the Strzelin Massif. Slightly modified from Oberc-Dziedzic et al. (2005).

rocks composed of plagioclase, quartz and microcline. The foliation is marked by biotite streaks. Muscovite plates are rare and oriented obliquely to the foliation. The gneisses contain sillimanite nodules up to $2 \mathrm{~cm}$ in diameter. The Nowolesie gneisses experienced two stages of the Variscan migmatization (Oberc-Dziedzic and Madej 2013).

\subsection{Gneisses of the Stachów Complex}

The pale Stachów orthogneisses (sensu lato) are divided into three groups: (i) the pale Stachów gneisses - sensu stricto, which form several meters thick tabular bodies in the dark Stachów paragneisses, often with poorly defined boundaries; (ii) the Henryków gneisses (Madej 1999), and (iii) the Gościęcice gneisses (Oberc-Dziedzic and Madej 2002), which both form large outcrops.

The pale Stachów gneisses (sensu stricto) are medium- or fine-grained rocks with streaky, lenticular or, less often, augen or banded texture. Augen of K-feldspar 1 to $2 \mathrm{~cm}$ long are rare. Layers and lenses composed of aggregates of quartz or aggregates of small grains of plagioclase and K-feldspar are surrounded by clusters of biotite and muscovite. Besides, two rare varieties have been observed, namely, fine-grained garnet-bearing gneisses and aplitic gneisses (Madej 2010).

The Henryków gneisses are strongly deformed rocks. They show a banded texture and the presence of 
Three generations of granitoids in the Strzelin Massif, implications for crustal evolution

K-feldspar porphyroclasts. They are composed of quartz, albite, chlorite and muscovite (Madej 1999, 2010).

The Gościęcice gneisses are coarse-grained, porphyritic rocks. Their granodioritic protolith has been deformed into augen gneisses with characteristic bluish, eye-shaped microcline grains up to $2 \mathrm{~cm}$ in diameter, often surrounded by plagioclase rims. More deformed gneisses show augen-banded and banded texture (Oberc-Dziedzic and Madej 2002).

\subsection{Variscan granitoids in the Strzelin Massif}

Based on ${ }^{206} \mathrm{~Pb} /{ }^{238} \mathrm{U}$ SHRIMP zircon ages (ObercDziedzic et al. 2010a, 2013a, 2015b; Oberc-Dziedzic and Kryza 2012), the Variscan igneous activity in the Strzelin Massif lasted for about $30 \mathrm{My}$, with three successive stages: I) tonalitic I (older) - c. 325 $\mathrm{Ma}$, II) granodioritic/granitic at c. $305 \mathrm{Ma}$ and III) tonalitic II (younger)/dioritic/granitic - from c. 295 to c. $280 \mathrm{Ma}$.

The first stage is documented by two small tonalite dykes (324 $\pm 4 \mathrm{Ma}$, Oberc-Dziedzic et al. 2010a) found in the borehole B1 (Bożnowice) (Fig. 2). The Bożnowice tonalite is a medium-grained rock composed of plagioclase, hornblende and biotite.

The second stage is represented by the Boznowice granodiorites from the southern part of the massif (306 $\pm 3 \mathrm{Ma}$, Oberc-Dziedzic et al. 2010a) and by medium-grained granites from the Strzelin I and II quarries (303 $\pm 2 \mathrm{Ma}$, Oberc-Dziedzic et al. 2013a, b) (Fig. 2). The Bożnowice granodiorite is a porphyritic rock, composed of quartz, perthitic microcline, plagioclase, parallel aligned plates of biotite and green hornblende. The medium-grained granite is a pale-grey rock composed of quartz, microcline, plagioclase and biotite. A faint lineation is defined by the alignment of feldspars and slightly elongated quartz aggregates.

The third stage corresponds to the most abundant granitoids, which have been further divided into three groups:

1) tonalites (II) and diorites (c. $295 \mathrm{Ma}$, ObercDziedzic et al. 2010a; Oberc-Dziedzic and Kryza 2012);

2) biotite-muscovite granites forming plutons (295 $\mathrm{Ma}$ ) and dykes cutting through the tonalites and biotite granites; their ages (283-295 Ma) are (nearly) the same as those of the enclosing granitoids (Oberc-Dziedzic et al. 2015b);

3) fine-grained biotite granites from the Strzelin quarries (283 $\pm 8 \mathrm{Ma}$, Oberc-Dziedzic et al. 2013a).

Tonalites II are fine- to medium-grained rocks. Medium-grained tonalites are composed of quartz,
Tab. 3 Chemical analyses of tonalites and diorites from the Strzelin Massif

\begin{tabular}{|c|c|c|c|c|c|c|}
\hline wt. \% & 4GT* & 5GT* & 9GT* & KL 3 & KC 1 & GR 2 \\
\hline $\mathrm{SiO}_{2}$ & 53.50 & 55.20 & 57.52 & 55.94 & 59.62 & 58.14 \\
\hline $\mathrm{TiO}_{2}$ & 1.64 & 1.28 & 0.91 & 1.25 & 1.07 & 0.83 \\
\hline $\mathrm{Al}_{2} \mathrm{O}_{3}$ & 17.95 & 16.45 & 17.68 & 16.80 & 16.63 & 13.46 \\
\hline $\mathrm{Fe}_{2} \mathrm{O}_{3}$ & 8.64 & 8.32 & 7.22 & 8.32 & 6.67 & 8.89 \\
\hline $\mathrm{Cr}_{2} \mathrm{O}_{3}$ & 0.01 & 0.02 & 0.00 & 0.01 & 0.01 & 0.08 \\
\hline $\mathrm{MnO}$ & 0.11 & 0.16 & 0.18 & 0.12 & 0.12 & 0.14 \\
\hline $\mathrm{MgO}$ & 4.37 & 4.47 & 3.36 & 3.90 & 2.95 & 7.83 \\
\hline $\mathrm{CaO}$ & 4.24 & 6.64 & 6.69 & 6.11 & 5.13 & 4.13 \\
\hline $\mathrm{Na}_{2} \mathrm{O}$ & 3.33 & 2.66 & 3.80 & 3.21 & 3.36 & 2.64 \\
\hline $\mathrm{K}_{2} \mathrm{O}$ & 4.07 & 2.12 & 1.46 & 2.56 & 3.02 & 2.34 \\
\hline $\mathrm{P}_{2} \mathrm{O}_{5}$ & 0.71 & 0.36 & 0.32 & 0.51 & 0.47 & 0.34 \\
\hline LOI & 1.05 & 1.70 & 0.70 & 1.20 & 0.90 & 1.10 \\
\hline Total & 99.62 & 99.38 & 99.84 & 99.93 & 99.95 & 99.92 \\
\hline $\mathrm{A} / \mathrm{CNK}$ & 1.00 & 0.90 & 0.90 & 0.90 & 0.90 & 0.90 \\
\hline $\mathrm{A} / \mathrm{NK}$ & 1.80 & 2.50 & 2.30 & 2.10 & 1.90 & 2.00 \\
\hline \multicolumn{7}{|l|}{$\mathrm{ppm}$} \\
\hline $\mathrm{Ba}$ & 879 & 401 & 317 & 601 & 561 & 468 \\
\hline $\mathrm{Rb}$ & 168 & 99 & 60 & 88 & 114 & 73 \\
\hline $\mathrm{Sr}$ & 286 & 353 & 310 & 334 & 340 & 246 \\
\hline $\mathrm{Y}$ & 21.8 & 24.7 & 35.6 & 27.2 & 34.0 & 26.9 \\
\hline $\mathrm{Zr}$ & 597.0 & 189.0 & 200.6 & 335.7 & 344.9 & 266.6 \\
\hline $\mathrm{Nb}$ & 33.4 & 24.6 & 18.3 & 26.7 & 29.3 & 21.4 \\
\hline Th & 7.0 & 2.9 & 5.5 & 8.3 & 11.7 & 10.3 \\
\hline $\mathrm{Ga}$ & 27.1 & 21.3 & 21.7 & 20.7 & 21.3 & 17.8 \\
\hline $\mathrm{Zn}$ & 128 & 136 & 84 & 81 & 92 & 52 \\
\hline V & 165 & 154 & 116 & 117 & 91 & 103 \\
\hline Hf & 11.2 & 4.1 & 5.2 & 8.3 & 8.2 & 6.4 \\
\hline $\mathrm{Tl}$ & 0.6 & 0.5 & 0.3 & 0.4 & 0.5 & 0.3 \\
\hline $\mathrm{Cu}$ & 38.0 & 49.0 & 11.8 & 6.1 & 3.7 & 129.5 \\
\hline $\mathrm{Pb}$ & 18.0 & 23.0 & 1.5 & 1.6 & 1.6 & 2.3 \\
\hline $\mathrm{Sn}$ & 8 & 19 & 7 & 4 & 9 & 2 \\
\hline Cs & 5.19 & 9.21 & 3.90 & 5.30 & 6.30 & 1.60 \\
\hline $\mathrm{Ta}$ & 2.3 & 1.4 & 1.3 & 2.1 & 2.0 & 1.6 \\
\hline $\mathrm{Ni}$ & 42.0 & 52.0 & 11.9 & 35.4 & 21.4 & 182.4 \\
\hline Co & 28.6 & 25.8 & 50.6 & 64.2 & 57.1 & 72.1 \\
\hline $\mathrm{U}$ & 10.4 & 1.5 & 1.6 & 2.8 & 2.4 & 1.9 \\
\hline $\mathrm{La}$ & 41.7 & 27.1 & 36.6 & 39.7 & 54.4 & 48.3 \\
\hline $\mathrm{Ce}$ & 79.0 & 59.5 & 94.0 & 91.5 & 128.4 & 105.6 \\
\hline $\operatorname{Pr}$ & 8.42 & 7.39 & 10.53 & 9.56 & 12.95 & 10.90 \\
\hline $\mathrm{Nd}$ & 30.6 & 29.9 & 40.8 & 36.2 & 49.3 & 41.6 \\
\hline Sm & 5.34 & 6.01 & 8.20 & 6.80 & 9.30 & 7.30 \\
\hline $\mathrm{Eu}$ & 1.29 & 1.58 & 1.88 & 1.70 & 2.09 & 1.57 \\
\hline $\mathrm{Gd}$ & 5.34 & 6.10 & 7.23 & 6.06 & 7.67 & 5.71 \\
\hline $\mathrm{Tb}$ & 0.72 & 0.85 & 1.18 & 0.93 & 1.16 & 0.92 \\
\hline Dy & 4.01 & 4.92 & 6.35 & 5.06 & 6.46 & 5.04 \\
\hline Ho & 0.78 & 0.94 & 1.20 & 0.97 & 1.14 & 0.92 \\
\hline $\mathrm{Er}$ & 2.30 & 2.65 & 3.39 & 2.94 & 3.31 & 2.49 \\
\hline $\mathrm{Tm}$ & 0.32 & 0.38 & 0.53 & 0.39 & 0.45 & 0.39 \\
\hline $\mathrm{Yb}$ & 2.10 & 2.37 & 3.12 & 2.36 & 2.87 & 2.44 \\
\hline $\mathrm{Lu}$ & 0.31 & 0.34 & 0.54 & 0.40 & 0.45 & 0.35 \\
\hline $\begin{array}{l}\mathrm{REE} \\
\end{array}$ & 182.2 & 150.0 & 215.6 & 204.6 & 280.0 & 233.5 \\
\hline $\mathrm{La}_{\mathrm{N}} / \mathrm{Yb}_{\mathrm{N}}$ & 14.2 & 8.2 & 8.4 & 12.1 & 13.6 & 14.2 \\
\hline $\mathrm{Eu} / \mathrm{Eu}^{*}$ & 0.74 & 0.80 & 0.75 & 0.81 & 0.76 & 0.74 \\
\hline
\end{tabular}

* - major-element analyses were published in Oberc-Dziedzic et al. (2015) 4GT, 5GT ,9GT (Gęsiniec q. N50²5'19.5", E17²'); KL 3 (N5043'47.7",

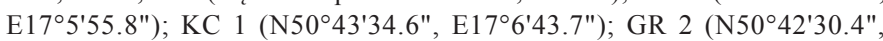
$\left.\mathrm{E} 17^{\circ} 7^{\prime} 10.8^{\prime \prime}\right)$ 

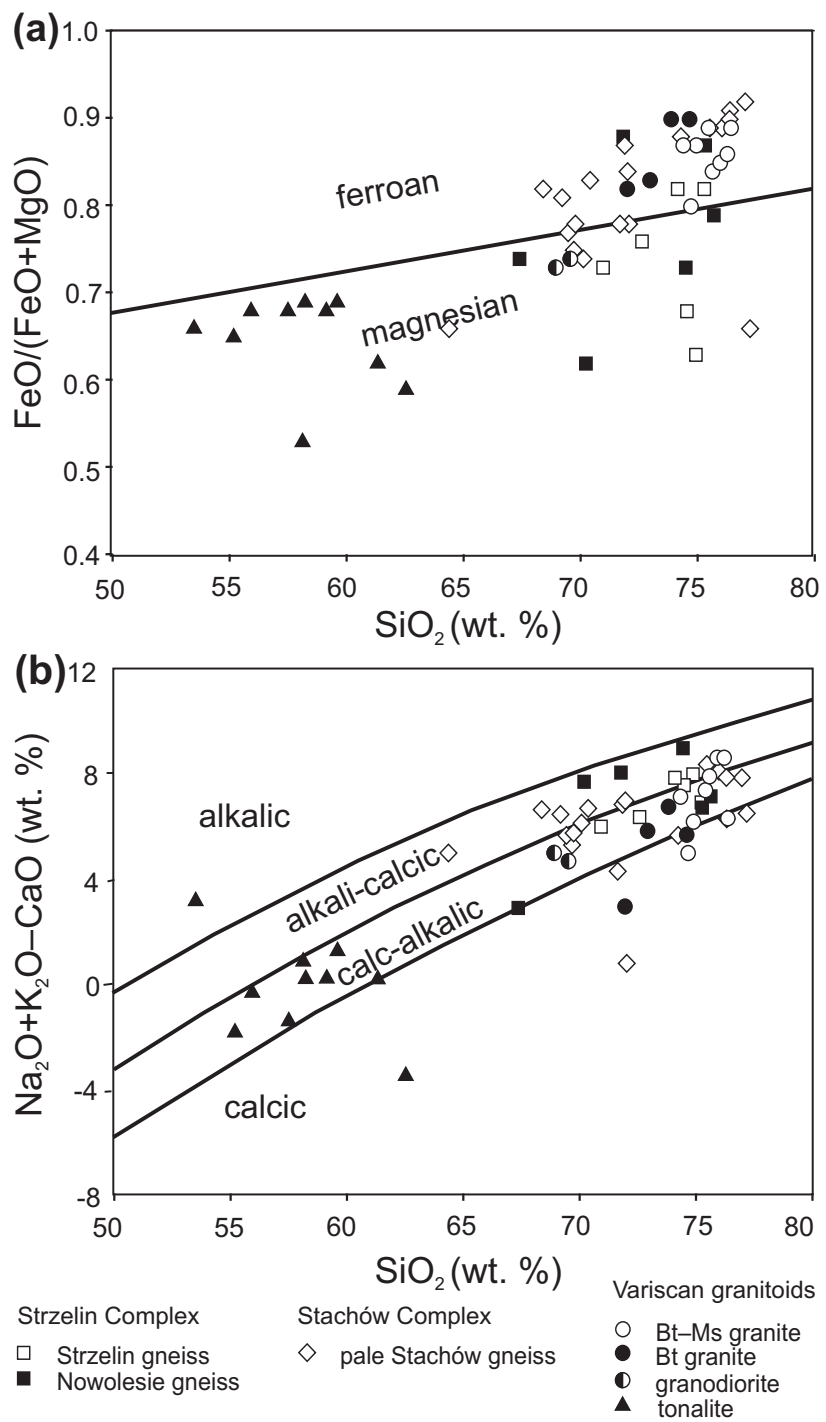

Fig. 4 Classification diagrams (Frost et al. 2001) for granitoids of the Strzelin Massif. a $-\mathrm{FeO} /(\mathrm{FeO}+\mathrm{MgO})$ vs. $\mathrm{SiO}_{2} \cdot \mathbf{b}-\mathrm{Na}_{2} \mathrm{O}+\mathrm{K}_{2} \mathrm{O}-\mathrm{CaO}$ vs. $\mathrm{SiO}_{2}$.

plagioclase, hornblende, biotite, apatite, titanite and opaque minerals (Pietranik and Waight 2008; Pietranik and Koepke 2009; Pietranik et al. 2006, 2009, 2011; Oberc-Dziedzic and Kryza 2012). The fine-grained tonalites locally contain relics of clinopyroxene and titaniteplagioclase-quartz clusters (Oberc-Dziedzic et al. 2010a).

Quartz diorites accompanying tonalites or forming separate, small intrusions are known from boreholes (e.g. GL 1, RG 2 - Fig. 2). These dark, medium- and finegrained rocks are composed of quartz, plagioclase, and grains or clusters of biotite and hornblende. Some quartz diorites contain relics of clinopyroxene or products of decomposition of pyroxene and hornblende. Textures and mineral composition of tonalites and diorites often vary inside the same magmatic body (Oberc-Dziedzic 2007; Pietranik and Waight 2008; Pietranik and Koepke 2009; Pietranik et al. 2006, 2009, 2011).
Fine-grained biotite-muscovite granites are composed of quartz, microcline, biotite, muscovite and some of them contain pinite (Oberc-Dziedzic and Pin 2000; Oberc-Dziedzic et al. 2015b).

The fine-grained biotite granite is composed of quartz, plagioclase, perthitic microcline and biotite, partly chloritized. It displays a faint lineation defined by the alignment of biotite (Oberc-Dziedzic et al. 2013a, b).

\section{Whole-rock geochemistry}

\subsection{Major elements}

The main types of orthogneisses and granitoids in the Strzelin Massif differ considerably in major-element composition (Tabs 1-3; ESM 1-4). The contents of $\mathrm{SiO}_{2}$ increase (rounded data, the highest and lowest values have been excluded) from the tonalites (53-62 wt. \%), through the Bożnowice granodiorite (68-70\%), the pale Stachów orthogneisses $(68-72 \%)$, the biotite granites (72-75\%), the Strzelin and Nowolesie gneisses (71$75 \%$ ), up to the biotite-muscovite granites (74-75\%).

The lowest contents of $\mathrm{K}_{2} \mathrm{O}(<4.0$ wt. $\%)$ are observed in the tonalites, and the highest (4.5-5.7 wt. \%) in the biotite-muscovite granites and the pale Stachów orthogneisses. The $600 \mathrm{Ma}$ gneisses (Strzelin and Nowolesie gneisses) differ from the rest of gneisses and granites by their higher contents of $\mathrm{Na}_{2} \mathrm{O}$ (usually more than 4.0 wt. \%) and lower contents of $\mathrm{CaO}$ (in general $<1.0$ wt. \%). The poorest in $\mathrm{Al}_{2} \mathrm{O}_{3}$ are the biotite and biotitemuscovite granites (12.8-13.8 wt. \%). Orthogneisses of the Stachów Complex contain more $\mathrm{Fe}_{2} \mathrm{O}_{3}, \mathrm{MgO}$, and $\mathrm{TiO}_{2}$ than gneisses of the Strzelin Complex and the Variscan granitoids.

Based on the classification of Frost et al. (2001), the tonalites, part of the Nowolesie gneisses and the granodiorites are magnesian whereas the Strzelin gneisses, part of the Nowolesie gneisses, the pale Stachów orthogneisses and the biotite and biotite-muscovite Variscan granites are ferroan (Fig. 4a). The tonalites, the Strzelin gneisses as well as the biotite and biotite-muscovite granites are situated in the calc-alkalic field, or close to it, in the calcic and alkali-calcic fields. Three samples of the Nowolesie gneisses are alkali-calcic (Fig. 4b). All the gneisses are peraluminous (Tabs 1-2; ESM 1-2). Granites are metaluminous to peraluminous (ESM 3), while tonalites are metaluminous (Tab. 3; ESM 4: Pietranik and Waight 2008).

\subsection{Trace elements}

The gneisses and granites of the Strzelin Massif differ from each other in $\mathrm{Ba}$ and $\mathrm{Sr}$ contents. The richest in 
$\mathrm{Ba}$ are the Bożnowice granodiorites (1500-1627 ppm; ESM 3), the poorest aplitic and garnet-bearing varieties of the pale Stachów gneisses (126-397 ppm; Tab. 2) and biotite-muscovite dyke granites (96-286 ppm; ESM 3). The $\mathrm{Sr}$ content is higher in the gneisses of the Strzelin Complex (214-479 ppm; Tab. 1; ESM 1) than in the orthogneisses of the Stachów Complex (99-275 ppm and 21-37 ppm in the aplitic variety; Tab. 2; ESM 2). Among Variscan granitoids, the $\mathrm{Sr}$ content diminishes from the Bożnowice granodiorites (600 ppm; ESM 3), through tonalites (246-334 ppm; Tab. 3; ESM 4), to biotite granites (141-184 ppm; ESM 3) and biotite-muscovite granites occurring as small plutons and dykes (78-117 and 21-63 ppm, respectively; ESM 3).

Noteworthy is the strong Y depletion in the Strzelin gneisses (4-9.6 ppm; Oberc-Dziedzic et al. 2005) and, to a lesser extent, in the Nowolesie gneisses. In contrast, the Stachów orthogneisses have much higher Y concentrations (23-80 ppm; Tab. 2). The Bożnowice granodiorite departs from the rest of the Variscan samples by remarkably low contents of Y (3-4 ppm; ESM 3).

The average Th contents are the lowest in the Strzelin and Nowolesie gneisses (3-7 ppm), higher in the pale Stachów gneisses (13-22 ppm; Tab. 2; ESM 2). The biotite and biotite-muscovite granites contain 11-21 ppm of Th (ESM 3) and tonalites 3-15 ppm (Tab. 3; ESM 4).

The trace-element contents of the studied gneisses and the Variscan granitoids were normalized to the average bulk continental crust composition of Rudnick and Gao (2005) (Fig. 5, left). Normalized abundances of many elements in the studied rocks (apart from cumulative Bożnowice granodiorites) are close to unity. Apart from the 600 Ma gneisses (the Strzelin and Nowolesie gneisses, Fig. 5a) and the plagioclase-cumulative Bożnowice granodiorites (Fig. 5e), all the samples show a negative $\mathrm{Sr}$ anomaly. More or less pronounced negative anomalies of Ti occur in all rocks but the tonalites, which have positive $\mathrm{P}$ anomaly (Fig. $5 \mathrm{~g}$ ). In the other rocks the $\mathrm{P}$ anomaly is either negative, or poorly expressed.

The studied rocks from the Strzelin Massif show variable total REE contents (Tabs 1-3; ESM 1-4). Overall, low $\Sigma$ REE occur in the Cadomian Strzelin and Nowolesie gneisses, the Early Ordovician garnet-bearing pale Stachów gneisses and the Variscan biotite-muscovite granites of dykes, irrespective of their ages.

On chondrite-normalized REE plots (Fig. 5, right), all granitoids apart from the Variscan tonalites and Neoproterozoic Strzelin gneisses show elevated LREE segment, relatively flat HREE segment, and a strong negative Eu anomaly, a pattern typical of many felsic igneous rocks. Three types of patterns can be distinguished, based on the mutual fractionation of the light and heavy REE, expressed as the $\mathrm{La}_{\mathrm{N}} / \mathrm{Yb}_{\mathrm{N}}$ ratio and the magnitude of the $\mathrm{Eu} / \mathrm{Eu}^{*}$ anomaly.
Type 1 is represented by the Strzelin gneisses and one sample of the Nowolesie gneiss, which show a pattern with strong and uniform fractionation from $\mathrm{La}$ to $\mathrm{Lu}$ (Fig. 5b). The $\mathrm{La}_{\mathrm{N}} / \mathrm{Yb}_{\mathrm{N}}$ ranges from 19 to 38, and both negative and positive $\mathrm{Eu} / \mathrm{Eu}^{*}(0.63-1.29)$ anomalies occur. The Nowolesie gneiss has more extreme characteristics, with $\mathrm{La}_{\mathrm{N}} / \mathrm{Yb}_{\mathrm{N}}=54$ and $\mathrm{Eu} / \mathrm{Eu}^{*}=1.72$, respectively (Tab. 1; ESM 1).

Type 2 is characterized by two-sectional distribution pattern of the REE, i.e. more or less steeply dipping LREE section and a nearly flat or gently dipping HREE section. Diagrams of the Type 2 were further divided into subtype $2 a$ and subtype $2 b$ on the basis of the $\mathrm{La}_{\mathrm{N}} / \mathrm{Yb}_{\mathrm{N}}$ ratio and the magnitude of the $\mathrm{Eu} / \mathrm{Eu}^{*}$ anomaly.

The subtype $2 a$ includes tonalites and, possibly, the Nowolesie gneisses. It shows a strong LREE enrichment with $\mathrm{La}_{\mathrm{N}}>100$ (Fig. 5b, h) and moderate fractionation of the LREE over the HREE, with $\mathrm{La}_{\mathrm{N}} / \mathrm{Yb}_{\mathrm{N}}$ ranging from 8.2 to 16.8 in tonalites (Tab. 3; ESM 4). Some of the Nowolesie gneisses (Fig. 5b) have REE patterns nearly parallel to those of tonalites (Fig. 5h), but their REE contents are much lower. Negative Eu anomalies occur in all samples of tonalites $\left(\mathrm{Eu} / \mathrm{Eu}^{*}=0.62-0.82\right.$; Tab. 3; ESM 4) and in the Nowolesie gneisses (0.52-0.87; Tab. 1).

Subtype $2 b$ includes the pale Stachów orthogneisses and the Variscan granites (Tab. 2; ESM 2-3; Fig. 5b, f). The highest $\mathrm{La}_{\mathrm{N}} / \mathrm{Yb}_{\mathrm{N}}$ ratios occur in the biotite granites (16 to 33), and they decrease in the biotite-muscovite granite from plutons (10 and 16), and especially in the biotite-muscovite granites from dykes (2.9 and 7.8). Both types of biotite-muscovite granites show a slight tetrad effect (Fig. 5f) (Irber 1999). In the Stachów pale orthogneisses, the $\mathrm{La}_{\mathrm{N}} / \mathrm{Yb}_{\mathrm{N}}$ ratios range from 4.3 to 9.5 . All the samples of group $2 \mathrm{~b}$ are characterized by strongly negative Eu anomalies (Tab. 2; ESM 2-3).

Type 3 corresponds to the Bożnowice granodiorites (Fig. 5f). They display distinct, steeply decreasing LREE and slightly increasing HREE branches, producing a saddle-shaped pattern. The $\mathrm{La}_{\mathrm{N}} / \mathrm{Yb}_{\mathrm{N}}$ ratios are 8.8-9.5 (ESM 3). Apart from the overall depletion, the most significant feature is a pronounced positive anomaly expressed by very high values of Eu/Eu* (7.5-9.0) (Fig. 5f, ESM 3).

\subsection{Sr-Nd isotopic data}

Bearing in mind the potentially significant mobility of $\mathrm{Rb}$ and Sr during deformation and metamorphism, only samples from the post-tectonic Variscan granites have been previously analysed for $\mathrm{Rb}-\mathrm{Sr}$ isotopes (Oberc-Dziedzic et al. 1996, 2015b; Oberc-Dziedzic and Pin 2000). The ${ }^{87} \mathrm{Sr} /{ }^{86} \mathrm{Sr}_{\mathrm{i}}$ ratios, age-corrected for the respective emplacement ages, constrained by the $\mathrm{U}-\mathrm{Pb}$ method on zircon, are listed in ESM 5. In general, relatively mildly radiogenic signatures are observed, specifically from 0.7065 

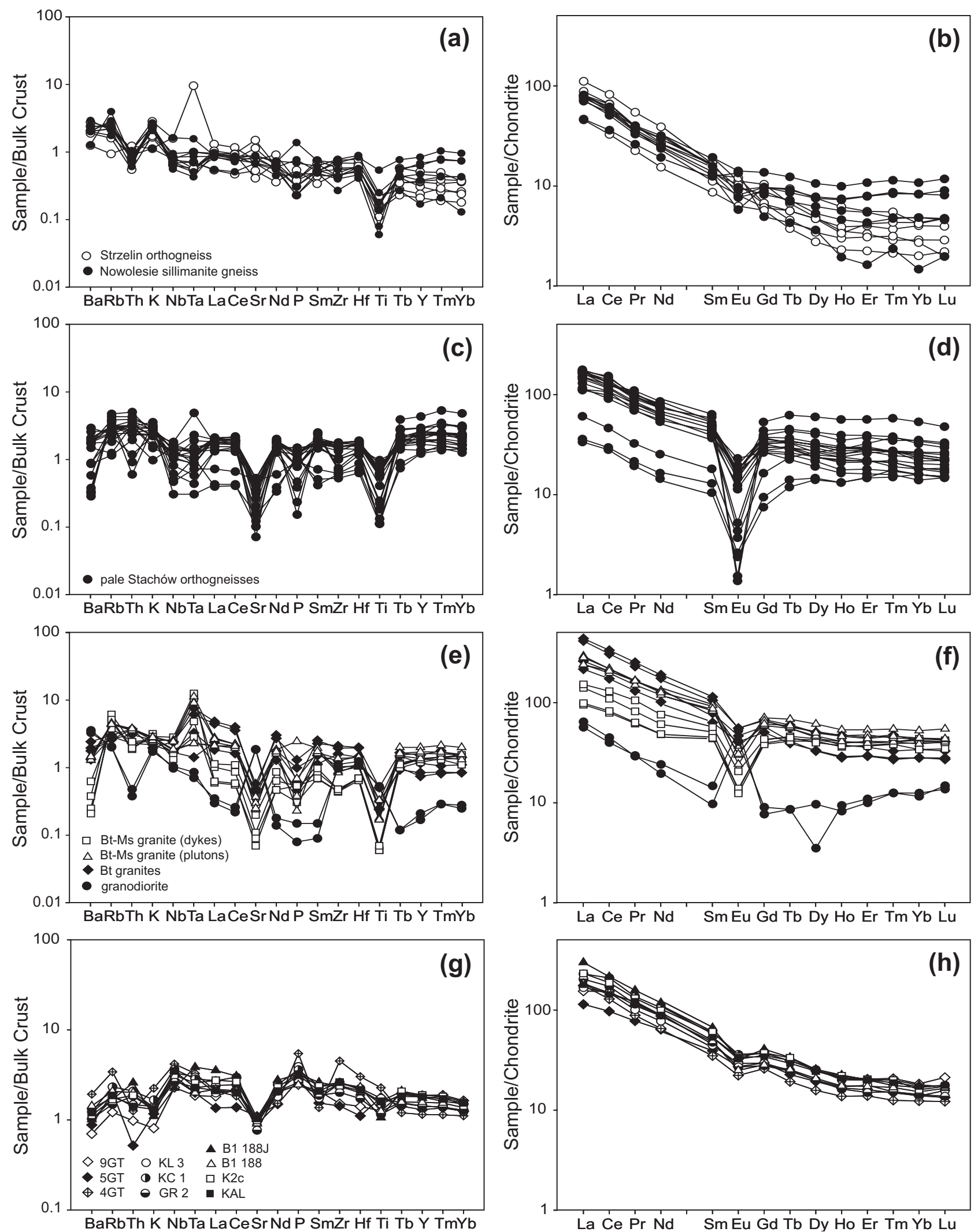

Fig. 5 Bulk continental crust normalized multielement plots (the left side; normalization values of Rudnick and Gao 2005) and chondrite-normalized REE plots (the right side; normalization values of Sun and McDonough 1989) for: a, b - the Neoproterozoic orthogneisses of the Strzelin Complex; c, $\mathbf{d}$ - the early Ordovician orthogneisses of the Stachów Complex; $\mathbf{e}, \mathbf{f}-$ the Variscan granites of the Strzelin Massif; $\mathbf{g}, \mathbf{h}-$ the Variscan tonalites and quartz diorites of the Strzelin Massif. 
Three generations of granitoids in the Strzelin Massif, implications for crustal evolution

Tab. $4 \mathrm{Sm}-\mathrm{Nd}$ isotope data for orthogneisses of the Strzelin Massif

\begin{tabular}{|c|c|c|c|c|c|c|c|c|c|c|}
\hline & Sample & Rock & $\mathrm{Sm}$ & $\mathrm{Nd}$ & ${ }^{147} \mathrm{Sm} /{ }^{144} \mathrm{Nd}$ & ${ }^{143} \mathrm{Nd} /{ }^{144} \mathrm{Nd}$ & $\varepsilon \mathrm{Nd}_{0}$ & $\varepsilon \mathrm{Nd}_{600 \mathrm{Ma}}$ & $\varepsilon \mathrm{Nd}_{500 \mathrm{Ma}}$ & $\mathrm{T}_{\mathrm{DM}}^{\mathrm{Nd}}(\mathrm{Ga})$ \\
\hline \multirow{7}{*}{ 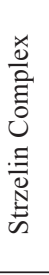 } & D 68-1 & Strzelin orthogneiss & 2.55 & 15.6 & 0.0991 & $0.511736(3)$ & -17.6 & -10.2 & & 1.74 \\
\hline & $210-50$ & Strzelin orthogneiss & 1.93 & 12.8 & 0.0910 & $0.511613(6)$ & -20.0 & -12.0 & & 1.78 \\
\hline & S 80 & Strzelin orthogneiss & 1.79 & 11.2 & 0.0970 & $0.511691(6)$ & -18.5 & -10.2 & & 1.77 \\
\hline & WL 2.11 & Strzelin orthogneiss & 1.58 & 9.5 & 0.1002 & $0.511736(3)$ & -17.6 & -10.3 & & 1.76 \\
\hline & SK 1 & Nowolesie sillimanite gneiss & 2.34 & 13.5 & 0.1048 & $0.511736(5)$ & -17.6 & -10.6 & & 1.83 \\
\hline & N 24 & Nowolesie sillimanite gneiss & 1.66 & 11.6 & 0.0864 & $0.511630(4)$ & -19.7 & -11.3 & & 1.69 \\
\hline & WL 3.15 & Nowolesie sillimanite gneiss & 2.49 & 14.6 & 0.1029 & $0.511692(3)$ & -18.5 & -11.3 & & 1.86 \\
\hline \multirow{6}{*}{ 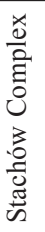 } & GD $10 / 3$ & Gościęcice orthogneiss & 5.55 & 27.8 & 0.1209 & $0.512047(5)$ & -11.6 & & -6.7 & 1.64 \\
\hline & $300 \mathrm{~V} 1$ & xenolith of pale orthogneiss & 6.25 & 27.7 & 0.1362 & $0.512235(9)$ & -7.9 & & -4.0 & 1.59 \\
\hline & $310 \mathrm{C}$ & Stachów pale orthogneiss & 5.68 & 24.7 & 0.1388 & $0.512196(6)$ & -8.7 & & -5.0 & 1.73 \\
\hline & WL 5.612 & Stachów pale orthogneiss & 7.10 & 35.0 & 0.1225 & $0.512117(5)$ & -10.2 & & -5.5 & 1.55 \\
\hline & WL-6.11 & Stachów pale orthogneiss & 2.12 & 9.8 & 0.1303 & $0.512129(3)$ & -10.0 & & -5.7 & 1.67 \\
\hline & HR 1.13 & Henryków orthogneiss & 6.65 & 33.1 & 0.1216 & $0.512144(3)$ & -9.7 & & -4.9 & 1.49 \\
\hline
\end{tabular}

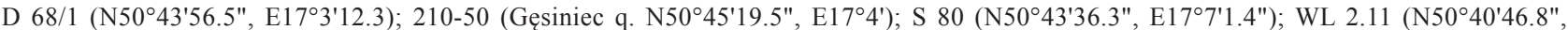

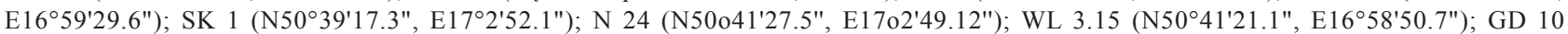

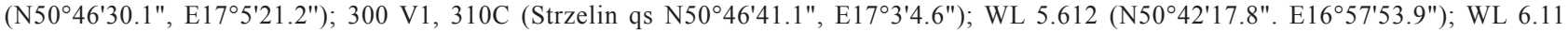
(N5043'23.4", E16 $\left.57^{\prime} 51.8 "\right)$; HR 1.13 (N50³8'41.9", E1659'51.2")

to $0.7079(0.7069 \pm 0.0005$, mean $\pm 1 \mathrm{sd}, \mathrm{n}=8)$ in the tonalites, from 0.7065 to $0.7080(0.7071 \pm 0.0006, \mathrm{n}=10)$ in the quartz diorites, and (discarding an outlying sample at 0.7098$)$ from 0.7062 to $0.7080(0.7072 \pm 0.0006, \mathrm{n}=$ $6)$ in the biotite granites.

Each rock type shows a certain amount of internal scatter, indicative that a significant degree of $\mathrm{Sr}$ isotope heterogeneity existed within each group. In the biotite granites, for example, the lowest ${ }^{87} \mathrm{Sr}^{86} \mathrm{Sr}_{\mathrm{i}}(0.7062)$ is found in the sample with the highest $\mathrm{Sr}$ content $(233$ $\mathrm{ppm}$ ) and the lowest $\mathrm{Rb} / \mathrm{Sr}$ ratio, while the highest ${ }^{87} \mathrm{Sr} /{ }^{86} \mathrm{Sr}$ initial ratios of 0.7080 and 0.7098 occur in the samples with much lower Sr contents (100 and 120 ppm, respectively).

The orthogneisses of the Strzelin Complex, of the Stachów Complex and the Variscan granitoids differ in their ${ }^{147} \mathrm{Sm} /{ }^{144} \mathrm{Nd}$ ratios (Tab. 4; ESM 6). The c. $600 \mathrm{Ma}$ gneisses of the Strzelin Complex have rather unusually low values ranging from 0.0864 to 0.1048 . Only the Bożnowice granodiorite $(306 \pm 3 \mathrm{Ma})$ displays similarly low value (0.0823), perhaps due to plagioclase accumulation. Higher ${ }^{147} \mathrm{Sm} /{ }^{144} \mathrm{Nd}$ ratios have been measured in the c. $500 \mathrm{Ma}$ orthogneisses of the Stachów Complex (0.1209-0.1388). The Variscan tonalites (c. $295 \mathrm{Ma})$ display ${ }^{147} \mathrm{Sm} /{ }^{144} \mathrm{Nd}$ ratios in the $0.1028-0.1213$ range, while the other rock types scatter between 0.1067 (finegrained, c. 283 Ma old biotite granite from the Strzelin I quarry, Oberc-Dziedzic et al. 2013a) and 0.1256 (biotite-muscovite granite dyke cutting the Gessiniec tonalite). Anomalously high $(0.1715-0.1804){ }^{147} \mathrm{Sm} /{ }^{144} \mathrm{Nd}$ ratios, associated to a $c$. threefold depletion in LREE were measured in the biotite-muscovite granite dykes from the Strzelin I quarry, and ascribed to the prior fractionation of (a) LREE-enriched phase(s) (ObercDziedzic et al. 2013a).
All the samples studied display age-corrected $\varepsilon \mathrm{Nd}_{i}$ values which are distinctly negative (Tab. 4; ESM 6) indicative of source regions that were depleted in $\mathrm{Sm}$ with regard to $\mathrm{Nd}$ on a time-integrated basis. The lowest values $\left(\varepsilon \mathrm{Nd}_{600}\right.$ from -10.2 to -12.0$)$ are found in the Neoproterozoic gneisses of the Strzelin Complex. Much higher, but still largely negative values occur in the c. 500 Ma pale Stachów orthogneisses $\left(-4.9<\varepsilon \mathrm{Nd}_{500}<-6.7\right)$. The sample of pale Stachów gneiss found as a xenolith in the Strzelin medium-grained biotite granite displays a $\varepsilon \mathrm{Nd}_{500}$ value of -4.0 , which might enlarge somewhat the -4.9 to -6.7 range, in so far as this did not undergo isotopic exchange with its less radiogenic $\left(\varepsilon_{28 d_{28}}=-3.8\right)$ host rock (ESM 6).

Tonalites show $\varepsilon \mathrm{Nd}_{300}$ values ranging from -2.8 to -4.3 (ESM 6; Pietranik and Waight 2008). The biotite-muscovite granites from the Gębczyce and Górka Sobocka quarries have $\varepsilon \mathrm{Nd}_{295}$ values of -4.5 and -4.8 , respectively. The biotite-muscovite granites of dykes are even less radiogenic: $\varepsilon \mathrm{Nd}_{283}-5.4$ and -5.7 for dykes from the Strzelin I quarry, and -5.9 for a dyke in the biotite-muscovite granite from the Gęsiniec quarry (ESM $6)$. The Bożnowice granodiorite shows the lowest epsilon value $\left(\varepsilon \mathrm{Nd}_{300}-7.7\right)$ among the Variscan granitoids from the Strzelin Massif.

The Depleted Mantle model ages ( $\mathrm{T}^{\mathrm{Nd}}$, DePaolo $1981 \mathrm{a}, \mathrm{b})$ used as a broad estimate of average crustal residence age for the source materials to the various granitoids, decrease systematically from the 600 Ma gneisses of the Strzelin Complex (1.69-1.86 Ga) through the pale Stachów orthogneisses $(1.49-1.73 \mathrm{Ga})$ to $1.09-1.46 \mathrm{Ga}$ of the Variscan granitoids (after rejection of the two samples with very high ${ }^{147} \mathrm{Sm} /{ }^{144} \mathrm{Nd}$ ratios, which obviously do not meet the basic requirements of the simplistic evolutionary model used in $\mathrm{T}_{\mathrm{DM}}^{\mathrm{Nd}}$ calculations). It is emphasized that 
these model ages do not convey any direct geological significance, but simply reflect the relative abundance of old recycled continental crustal materials in the studied granitoids.

\section{Inherited zircon cores}

The three generations of granitoids contain zircons with inherited cores which can put constraints on the source material for granitoid magmas.

The Neoproterozoic Strzelin and Nowolesie gneisses of the Strzelin Complex, both c. 600 Ma old, contain inherited cores with ${ }^{207} \mathrm{~Pb} /{ }^{206} \mathrm{~Pb}$ ages (providing minimum estimates of their initial crystallization event) ranging between c. 2000 and $1240 \mathrm{Ma}$ in the Strzelin gneiss (ObercDziedzic et al. 2003) and between c. 2060 and $1510 \mathrm{Ma}$ (Klimas 2008; Klimas et al. 2009) or c. 1750 to 1200 (Mazur et al. 2010) in the Nowolesie gneiss from Skalice.

In the c. $500 \mathrm{Ma}$ orthogneisses of the Stachów Complex (Oliver et al. 1993; Klimas 2008; Mazur et al. 2010), inherited cores yield ages of $1.6-2.9 \mathrm{Ga}\left({ }^{207} \mathrm{~Pb} /{ }^{206} \mathrm{~Pb}\right.$ ages) and c. $590 \mathrm{Ma}\left({ }^{206} \mathrm{~Pb} /{ }^{238} \mathrm{U}\right.$ age) (Mazur et al. 2010). The presence of inherited zircon cores of Paleo- to Neoproterozoic ${ }^{206} \mathrm{~Pb} /{ }^{238} \mathrm{U}$ ages of $1.91 \mathrm{Ga}, 640$ and $560 \mathrm{Ma}$ was also mentioned by Klimas (2008).

Zircons from the Variscan granitoids in the Strzelin Massif contain much less inherited cores than those from the orthogneisses. In the tonalites and the Strzelin medium-grained biotite granite, inherited cores are indeed conspicuously absent (Oberc-Dziedzic et al. 2010a, 2013a; Oberc-Dziedzic and Kryza 2012). In the other granitoids, i.e. the Bożnowice granodiorite, the Strzelin fine-grained biotite granite, biotite-muscovite granites of both plutons and dykes, inherited zircon cores occur, with ages fairly similar to those reported for the orthogneisses. Interestingly, in cases the zircon cores have the same ages as zircons from their country-rocks, e.g. the Stachów gneisses for the Strzelin fine-grained biotite granite, the Strzelin Complex gneisses for the Bożnowice granodiorite, and the biotite-muscovite granite dyke cutting the Gęsiniec tonalite, respectively.

\section{Discussion of relations between three groups of granitoids in the Strzelin Massif}

\subsection{The bearing of geochemical data}

\subsubsection{Trace and major elements}

In the three age groups of granitoids, trace-element (including the REE) abundances are close to the bulk con- tinental crust estimates (Rudnick and Gao 2005; Fig. 5, left). Moreover, the trace-element data in all the granitoid groups display typical continental crustal features (high contents of $\mathrm{K}$ and $\mathrm{Rb}$, LREE enrichment, pronounced negative anomalies in Eu and in HFSE). The Neoproterozoic Strzelin gneisses are peculiar in lacking Sr negative anomaly, and by a sodic character. Although their chondrite-normalized LREE patterns are steep (Fig. 5b), they are also poorer in the most incompatible elements than the other granitoids and their HREE are fractionated in most cases, suggesting the persistence of residual garnet in their source. The tonalites also depart from the rest of granitoids, with a pronounced LREE enrichment associated with insignificantly negative Eu anomalies. Together with their lower $\mathrm{SiO}_{2}$ and higher $\mathrm{CaO}, \mathrm{Fe}_{2} \mathrm{O}_{3}$ and $\mathrm{MgO}$ contents, these features indicate that different sources (i.e. mafic lower crust or mantle-derived magmas) were involved in their genesis.

The influence of the magma differentiation on the concentration of some trace elements is well visible in the aplitic variety of the pale Stachów orthogneisses, distinctly depleted in many elements (Tab. 2) but enriched in $\mathrm{Y}$, thereby suggesting that the HREE behaved as incompatible elements throughout the igneous evolution. Similarly, the negative anomalies in $\mathrm{Nb}, \mathrm{Sr}, \mathrm{P}$ and $\mathrm{Ti}$ of the gneisses and granites may reflect fractionation of plagioclase, apatite, and $\mathrm{Fe}-\mathrm{Ti}$ oxides during earlier stages of magma evolution. On the contrary, the pronounced positive Eu anomaly of the Bożnowice granodiorites $\left(\mathrm{Eu} / \mathrm{Eu}^{*}=7.5-9.0\right.$ : Fig. $\left.5 \mathrm{f}\right)$, indicates that this rock contains substantial amounts of cumulus feldspar, also clearly reflected by the high $\mathrm{Sr}$ and $\mathrm{Ba}$, as well as a general depletion (by dilution) of all trivalent lanthanides (Oberc-Dziedzic et al. 2010a). The contrasting chondritenormalized REE patterns (e.g. the Strzelin and Nowolesie gneisses, Fig. 5b) may reflect various petrogenetic processes (e.g. different source compositions, more intense partial melting or less evolved fractionation).

Summing up, the trace-element data provide important arguments concerning the crustal affinity of three groups of granitoids of different age, and similarity of some petrogenetic processes, but they do not allow resolving their mutual relationships.

\subsubsection{Radiogenic isotopic ratios}

The initial ${ }^{87} \mathrm{Sr} /{ }^{86} \mathrm{Sr}$ ratios of tonalites, quartz diorites and granites are scattered (ESM 5). This can be interpreted to reflect either isotopic variations in the source protoliths, or the influence of relatively late-stage disturbances, namely an open-system behavior involving the introduction of radiogenic crustal fluids or partial melts, or both. It can be seen, however, that the mean values of ${ }^{87} \mathrm{Sr} /{ }^{86} \mathrm{Sr}$ initial ratios in each rock-type display 
a slight increase from tonalites $(0.7069)$ to biotite granites $(0.7072)$ through quartz diorites $(0.7071)$, suggesting that combined assimilation-fractional crystallization (AFC) involving the assimilation of extraneous ${ }^{87} \mathrm{Sr}$-rich components may have played some role in the petrogenesis of the Variscan granitoid suite. This interpretation is further supported by the observation that the more evolved biotite-muscovite granites display even more radiogenic signatures (from 0.7086 to 0.7104 ), reaching values as high as 0.718 and 0.726 in Sr-poor (20-30 ppm) aplitic samples. In this case, it is possible that crustal aqueous fluids carrying highly radiogenic $\mathrm{Sr}$ strongly contaminated the last volumes of residual melt left after extensive fractionation.

The negative Nd epsilon values document a long-term enrichment of Nd over Sm in the source reservoirs. Geologically, this feature is interpreted to reflect the dominant role of geochemically evolved crustal components in the production of granitic magmas, generated from much older, LREE-enriched continental crust sources. Good example are the Bożnowice granodiorites which are REE-poor and show the lowest $\varepsilon \mathrm{Nd}_{300}$ value (-7.7) found among the Variscan granitoids. Their parental magma would have been fairly sensitive to contamination by ancient crustal components with unradiogenic $\mathrm{Nd}$ isotope signature, such as the Neoproterozoic Strzelin gneisses $\left(\varepsilon \mathrm{Nd}_{300}=-14\right.$ to -16$)$.

The $\varepsilon \mathrm{Nd}_{\mathrm{i}}$ values of the fine-grained biotite granite and tonalites (ESM 6) are higher (less negative) than those from gneisses, granodiorites and the biotite-muscovite granites. This documents magma sources that were somewhat less enriched in LREE on a time-integrated basis, or that they were younger, or both. Possibly, this might reflect the involvement of a subordinate contribution of a mafic (mantle-derived) material at the time of magma generation.

Although the Depleted Mantle Nd model ages $\left(\mathrm{T}_{\text {DM }}^{\mathrm{Nd}}\right.$, Tab. 4; ESM 6) used as a broad estimate of crustal residence age, do not convey any straightforward geological information, they emphasize the major role for recycling of preexisting continental crustal materials in the production of all the granitoids of the Strzelin Massif. This is valid irrespective of their age. However, there is also a general tendency of increasing $\varepsilon \mathrm{Nd}_{\mathrm{i}}$ values with decreasing age, which is accompanied by decreasing average crustal residence ages of the source materials of the particular groups of gneisses and granitoids of the Strzelin Massif. This shows that materials with higher time-integrated $\mathrm{Sm} / \mathrm{Nd}$ ratios played an increasing role during the successive events of granitoid generation.

From a petrogenetic point of view, this observation might be interpreted to reflect successive partial melting episodes of a single crustal source which would become increasingly refractory as a result of successive extrac- tion events of granitic melts. In this scenario, the crustal source would be stripped of its most fusible components during the first partial melting episode, with a concomitant increase of the $\mathrm{Sm} / \mathrm{Nd}$ ratio in the residue. Accordingly, the subsequent fusion would involve a relatively more mafic and refractory source, thereby requiring a higher heat input to overcome the lower fertility. It would be probably associated with the advection of mantle magmas which may have also contributed to magma production through hybridization.

Alternatively, an isotopically zoned crustal segment composed of a pile of fertile materials could be envisioned, with an older more felsic lower part (e.g. sediments ultimately dominated by Eburnean, c. $2 \mathrm{Ga}$ sources) overlain by a younger and/or more mafic (i.e. isotopically more juvenile) layer (e.g. Neoproterozoic arc-related lavas and pyroclastic rocks). This heterogeneous crust would have suffered successive partial melting episodes over the c. 300 My time span recorded in the Strzelin Massif.

\subsection{The meaning of inherited zircon-core ages}

The age patterns of inherited zircon cores provide important constraints for drawing inferences on possible relationships between the different granitoids. The inherited zircon-core ages from the Neoproterozoic Strzelin and Nowolesie gneisses fall between c. 2.0 and $1.2 \mathrm{Ga}$ (Oberc-Dziedzic et al. 2003; Klimas 2008; Klimas et al. 2009; Mazur et al. 2010). This wide range of inherited ages indicates that several different zircon-bearing (that is, relatively felsic) crustal sources were ultimately involved in the production of the magmas parental to the Strzelin and Nowolesie orthogneisses (Oberc-Dziedzic et al. 2003). This range further suggests a detrital sedimentary origin for the source rocks which were partially melted c. 600 Ma ago.

Accordingly, it is probable that multiple recycling occurred during the evolution of these protoliths, thereby precluding any simple geological implications to be drawn. It is noticed that the inherited zircon cores age spectra from the Strzelin and Nowolesie gneisses resemble those considered as typical of Avalonia (Friedl et al. 2000). The ages of inherited zircons from the Early Ordovician Stachów orthogneisses range between 1640 and $2910 \mathrm{Ma}$ and c. 560-640 Ma (Klimas 2008; Mazur et al. 2010), typical of Armorica (Friedl et al. 2000) and similar to those found in coeval orthogneisses of the Orlica-Śnieżnik Dome (Turniak et al. 2000; Kröner et al. 2001) or in the Karkonosze-Izera Massif (Kröner et al. 2001; Oberc-Dziedzic et al. 2009; Żelaźniewicz et al. 2009). These contrasting inherited zircon-core data from orthogneisses of the Stachów and Strzelin Complexes 
thus suggest that their protoliths originated from different sources, as also indicated by their contrasting $\mathrm{Nd}$ isotope signatures.

The Variscan granitoids contain much less inherited zircon cores than orthogneisses, either because their source materials contained less zircon, or because the parental magmas were not saturated in zircon due to their relatively Si-poor, metaluminous chemistry.

The similarity of the zircon-core ages in some Variscan granitoids of the Strzelin Massif, and in zircons from their gneissic country rocks may suggest a genetic relationship (e.g. the Bożnowice granodiorite and biotitemuscovite granites with the Neoproterozoic gneisses) but it could also merely imply that these ancient zircons are not inherited cores sensu stricto (that is, derived from the source and present in the granitic magma from its inception), but rather xenocrysts incorporated in the magma during its ascent and emplacement.

\subsection{What do the Strzelin Massif granitoids tell about the development of the local continental crust?}

The geochemical and $\mathrm{Nd}$ isotope data point to the largely crustal origin of the three diachronous groups of granitoids in the Strzelin Massif. This conclusion is in agreement with a designation of the $600 \mathrm{Ma}$ Strzelin and Nowolesie orthogneisses, the 500 Ma pale Stachów orthogneisses and the Variscan biotite-muscovite granites as S-type granitoids (Oberc-Dziedzic et al. 2005). This is based on the lack of mafic enclaves and the broadly homogeneous and monotonous mineralogical composition of these rocks, with quartz, K-feldspar, relatively sodic plagioclase, primary muscovite (Oberc-Dziedzic et al. 2015b) and biotite. The S-type character of the 600 $\mathrm{Ma}$ and $500 \mathrm{Ma}$ gneisses and the Variscan biotite-muscovite granites is also documented by their peraluminous character, and the occurrence of several generations of inherited zircons. The metaluminous chemistry of biotite granites and the presence of the mafic microgranular enclaves in them (Lorenc 1984), point to their I-type affinity. A similar affinity is shown by tonalites and diorites (Pietranik and Waight 2008; Pietranik and Koepke 2009; Pietranik et al. 2006, 2011; Oberc-Dziedzic 2007).

The average contents of the major and trace elements in the three groups of granitoids of the Strzelin Massif exclude the possibility that the younger granitoids were formed by partial melting of the older ones. Such a scenario would suggest that the magma of three granitoids has evolved in a sequence reflecting increasing $\mathrm{SiO}_{2}$ and diminishing contents of, for instance, $\mathrm{Mg}$, Fe, which is at odds with the observations (Tabs 1-3; ESM 1-4).

Good arguments concerning relations between three groups of granitoids come from ages of inherited zircon cores. The different inherited-core age spectra exclude close relations between the $c$. 500 Ma pale Stachów orthogneisses and the $c$. $600 \mathrm{Ma}$ Strzelin and Nowolesie gneisses, while similar inherited zircon ages in the 600 Ma gneisses and the Variscan granites might suggest that these two groups of granitoids were generated from common, or at least broadly similar source(s). However, a comparison of the $\varepsilon \mathrm{Nd}$ values of the $c .600 \mathrm{Ma}$ old gneisses calculated as they were $300 \mathrm{Ma}$ ago $\left(\varepsilon \mathrm{Nd}_{300}\right.$ from -14 to -16 , Oberc-Dziedzic et al. 2015b) and the $\varepsilon_{300}$ values of the fine-grained biotite, biotite-muscovite granite and granodiorite $(-3.8,-4.5$ to -5.9 and -7.7 respectively) invalidates such a simple interpretation and reinforces the conclusion that these gneisses could have contributed only marginally, if at all, to the production of the Variscan granitoids (Oberc-Dziedzic et al. 2015b). If the $600 \mathrm{Ma}$ gneisses and the Variscan granites originated from a common lower crustal source, then a significant contribution of mantle-derived magmas is required to the production of the Variscan granitoids (Pietranik and Waight 2008; Pietranik and Koepke 2009).

\section{Conclusions}

1) In the Strzelin Massif, three groups of (meta-)granitoids occur: (i) the Neoproterozoic (c. $600 \mathrm{Ma}$ ) Strzelin and Nowolesie orthogneisses of the Strzelin Complex; (ii) the Early Ordovician (c. $500 \mathrm{Ma}$ ) orthogneisses of the Stachów Complex (the pale Stachów gneisses); (iii) the Variscan granitoids represented by the Bożnowice granodiorite as well as the Strzelin mediumand fine-grained biotite granites, biotite-muscovite granites and tonalities-diorites.

2) The trace element data provide important arguments concerning the crustal affinity of three groups of granitoids of different age, and similarity of some petrogenetic processes, but they do not constrain their mutual relationships.

3) The contrasting $\varepsilon \mathrm{Nd}$ values of the $600 \mathrm{Ma}$ gneisses and the Variscan granitoids clearly document that the continental crust of the massif was enriched in isotopically more juvenile, mantle-derived material during Carboniferous.

4) The general tendency of increasing $\varepsilon \mathrm{Nd}_{i}$ values with decreasing geological age concurs with decreasing average crustal residence ages $\left(\mathrm{T}_{\mathrm{DM}}^{\mathrm{Nd}}\right)$ of the individual groups of (meta-) granitoids in the Strzelin Massif. Although the 500 Ma gneisses are allochtonous in the massif, their $\varepsilon \mathrm{Nd}_{\mathrm{i}}$ values fit very well to the trend: the younger the granite, the less negative its $\varepsilon \mathrm{Nd}_{\mathrm{i}}$ value.

5) The differences in inherited zircon ages observed between the Early Ordovician gneisses of the allochtonous Stachów Complex (similar to the ages spectra 
found in coeval orthogneisses from the West Sudetes, and believed to be of Armorica type), and the Neoproterozoic gneisses of the para-autochtonous Strzelin Complex (considered as typical of Avalonia), suggest that they did not have a common ancestry.

Acknowledgments. The investigations were performed within the Project N307 008 32/0314 of the Ministry of Science and Higher Education. Comments on the manuscript by A. Gawęda and two anonymous reviewers helped to refine the manuscript. The authors are very grateful to handling editor J. Žák for his patience. The extremely careful editorial corrections of V. Janoušek significantly improved the manuscript.

Electronic supplementary material. The published wholerock geochemical and $\mathrm{Sr}-\mathrm{Nd}$ isotopic analyses from the Strzelin Massif as well as GPS coordinates of the studied samples are available online at the Journal web site (http://dx.doi.org/10.3190/jgeosci.227).

\section{References}

Aleksandrowski P, Mazur S (2002) Collage tectonics in the northeasternmost part of the Variscan Belt: the Sudetes, Bohemian Massif. In: Winchester JA, Pharaoh TC, Verniers J (eds) Palaeozoic Amalgamation of Central Europe. Geological Society of London Special Publications 201: 237-277

Awdankiewicz M, Awdankiewicz H, Kryza R, Rodionov N (2010) SHRIMP zircon study of a micromonzodiorite dyke in the Karkonosze Granite, Sudetes (SW Poland): age constraints for late Variscan magmatism in Central Europe. Geol Mag 147: 77-85

Badura J (1979) Detailed map of the Sudetes, map sheet Stolec $1: 25,000$. Geological Institute, Wydawnictwa Geologiczne, Warsaw (in Polish)

Bialek D, Kryza R, Oberc-Dziedzic T, Pin C (2014) Cambrian Zawidów granodiorites in the Cadomian Lusatian Massif (Central European Variscides): what do the SHRIMP zircon ages mean? J Geosci 59: 313-326

BorkowsKa M, HameurT J, VidAL P (1980) Origin and age of Izera gneisses and Rumburk granites in the Western Sudetes. Acta Geol Pol 30: 121-146

De Paolo J (1981a) Neodymium isotopes in the Colorado Front Range and crust - mantle evolution in the Proterozoic. Nature 291: 193-196

De Paolo J (1981b) A Nd and Sr isotopic study of Mesozoic calc-alkaline batholiths of the Sierra Nevada and Peninsular Ranges, California. J Geophys Res 86: 10370-10488

Dörr W, Żelaźniewicz A, Bylina P, Schastok J, Franke W, HAACK U, Kulicki C (2006) Tournaisian age of grani- toids from the Odra Fault Zone (southwestern Poland): equivalent of the Mid-German Crystalline High? Int J Earth Sci (Geol Rundsch) 95: 341-349

DudEK A (1980) The crystalline basement block of the Outer Carpathians in Moravia: Bruno-Vistulicum. Rozpr Čs Akad Věd, ̌̌ mat přír Věd 90: 1-85

Finger F, Hanžl P, Pin C, Von Quadt A, Steyrer HP (2000) The Brunovistulian: Avalonian Precambrian sequence at the eastern end of the Central European Variscides? In: Franke W, Haak V, Oncken O, Tanner D (eds) Orogenic Processes: Quantification and Modelling in the Variscan Belt. Geological Society of London Special Publications 179: 103-112

FrANKE W (1989) Variscan plate-tectonics in Central Europe - current ideas and open questions. Tectonophysics 169: $221-228$

FRANKE W (2000) The mid-European segment of the Variscides: tectonostratigraphic units, terrane boundaries and plate tectonic evolution. In: Franke W, HAAK V, ONCKEN O, TANNer D (eds) Orogenic Processes: Quantification and Modelling in the Variscan Belt. Geological Society of London Special Publications 179: 35-61

Franke W (2012) Comment on Dörr and Zulauf: Elevator tectonics and orogenic collapse of a Tibetan-style plateau in the European Variscides: the role of the Bohemian shear zone. Int J Earth Sci (Geol Rundsch) 101: 2027-2034

Franke W, ŻELAŹNIEWICZ A (2000) The eastern termination of the Variscides: terrane correlation and kinematic evolution. In: Franke W, HaAK V, OnCKen O, TANNER D (eds) Orogenic Processes: Quantification and Modelling in the Variscan Belt. Geological Society of London Special Publications 179: 63-86

Friedl G, Finger F, McNaughton NJ, Flechter IR (2000) Deducing the ancestry of terranes: SHRIMP evidence for South America-derived Gondwana fragments in central Europe. Geology 28: 1035-1038

Frost BR, Barnes CG, Collins WJ, Arculus RJ, Ellis DJ, Frost CD (2001) A geochemical classification for granitic rocks. J Petrol 11: 2033-2048

IRBER W (1999) The lanthanide tetrad effect and its correlation with $\mathrm{K} / \mathrm{Rb}, \mathrm{Eu} / \mathrm{Eu}^{*}, \mathrm{Sr} / \mathrm{Eu}, \mathrm{Y} / \mathrm{Ho}$, and $\mathrm{Zr} / \mathrm{Hf}$ of evolving peraluminous granite suites. Geochim Cosmochim Acta 63: 489-508

JACOBSEN SB, WASSERBURG GJ (1980) Sm-Nd isotopic evolution of chondrites. Earth Planet Sci Lett 50: 139-155

Kemp AJS, Hawkesworth CJ (2005) Granitic perspectives on the generation and secular evolution of the continental crust. In: RuDNICK RJ (ed) The Crust. Treatise on Geochemistry, Vol. 3. Elsevier-Pergamon, Oxford, pp 1-64

KLIMAS K (2008) Geochronology and Petrogenetical Study of Zircons from Selected Crystalline Rocks in the Eastern Part of the Fore-Sudetic Block. University of Wrocław 
Institute of Geological Sciences, Wrocław, pp 1-194 (in Polish, English summary)

Kuimas K, Kryza R, Fanning CM (2009) Palaeo- to Mesoproterozoic inheritance and Ediacaran anatexis recorded in gneisses at the NE margin of the Bohemian Massif: SHRIMP zircon data from the Nowolesie gneiss, ForeSudetic Block (SW Poland). Geol Sudetica 41: 25-40

Kossmat F (1927) Gliederung des varistischen Gebirgsbaues. Abh Sächs Geol Landesamts 1: 1-39

Kröner A, Jaeckel P, Hegner E, Opletal M (2001) Single zircon ages and whole-rock $\mathrm{Nd}$ isotopic systematics of early Palaeozoic granitoid gneisses from the Czech and Polish Sudetes (Jizerské hory, Krkonoše Mountains and Orlice-Sněžník Complex). Int J Earth Sci (Geol Rundsch) 90: 304-324

Kryza R, Crowley QG, Larionov A, Pin C, Oberc-Dziedzic T, MOCHNACKA K (2012) Chemical abrasion applied to SHRIMP zircon geochronology: an example from the Variscan Karkonosze Granite (Sudetes, SW Poland). Gondwana Res 21: 757-767

Kryza R, Schaltegger U, Oberc-Dziedzic T, Pin C, OvtchaROVA M (2014a) Geochronology of a composite granitoid pluton: a high-precision ID-TIMS U-Pb zircon study of the Variscan Karkonosze Granite (SW Poland). Int J Earth Sci (Geol Rundsch) 103: 683-696

Kryza R, Pin C, Oberc-Dziedzic T, Crowley QG, Larionov A (2014b) Deciphering the geochronology of a large granitoid pluton (Karkonosze Granite, SW Poland): an assessment of $\mathrm{U}-\mathrm{Pb}$ zircon SIMS and $\mathrm{Rb}-\mathrm{Sr}$ whole-rock dates relative to $\mathrm{U}-\mathrm{Pb}$ zircon CA-ID-TIMS. Inter Geol Rev 56: 756-782

Kusiak MA, Dunkley DJ, SŁaby E, Martin H, Budzyń B (2009) Sensitive high-resolution ion microprobe analysis of zircon reequilibrated by late magmatic fluids in a hybridized pluton: Geology 37: 1063-1066

Kusiak MA, Williams I, Dunkley DJ, Konečný P, SŁaby E, Martin H (2014) Monazite to the rescue: U-Th-Pb dating of the intrusive history of the composite Karkonosze pluton, Bohemian Massif: Chem Geol 364: 76-92

Laurent A, Janoušer V, Magna T, Schulmann K, Míková J (2014) Petrogenesis and geochronology of a postorogenic calc-alkaline magmatic association: the Žulová Pluton, Bohemian Massif. J Geosci 59: 415-440

LORENC MW (1984) Endogenous enclosures (autoliths) as evidence of magmatic origin of the Strzelin granitoids. Geol Sudetica 19: 75-97 (in Polish, English summary)

Machowiak K, Armstrong R (2007) SHRIMP U-Pb zircon age from the Karkonosze granite. Miner Pol Spec Pap 31: 193-196

MAdEJ S (1999) Extensional tectonics in the eastern part of the Fore-Sudetic Block: evidence from the Henryków gneiss. Miner Soc Pol Spec Pap 14: 38-41

Madej S (2010) Petrology and tectonics of gneisses from the Lipowe Hills (the Fore-Sudetic Block). Unpublished
Ph.D. thesis, University of Wrocław, pp 1-259 (in Polish)

Matte P, Maluski H, Rajlich P, Franke W (1990) Terrane boundaries in the Bohemian Massif: results of large-scale Variscan shearing. Tectonophysics 177: 151-170

Mazur S, Aleksandrowski P, Kryza R, Oberc-Dziedzic $\mathrm{T}$ (2006) The Variscan Orogen in Poland. Geol Q 50: $89-118$

Mazur S, Kröner A, Szczepański J, Turniak K, HanžL P, Melichar R, Rodionov NV, Paderin I, Sergeev SA (2010) Single zircon U-Pb ages and geochemistry of granitoid gneisses from SW Poland: evidence for an Avalonian affinity of the Brunian microcontinent. Geol Mag 147: 508-526

McLennan SM, TaYlor SR, Hemming SR (2006) Composition, differentiation, and evolution of continental crust: constraints from sedimentary rocks and heat flow. In: Brown M, Rushmer T (eds) Evolution and Differentiation of the Continental Crust. Cambridge University Press, Cambridge, New York, Melbourne, pp 92-134

OBERC J (1966) Geology of crystalline rocks of the Wzgórza Strzelińskie Hills, Lower Silesia. Studia Geol Pol 20: 1-187 (in Polish, English summary)

OBERc-DzieDzic T (2007) Internal structure of the granite and tonalite intrusions in the Strzelin Massif. In: KozŁowsKI A, Wiszniewska J (eds) Granitoids in Poland. Archivum Mineralogiae Monograph 1. University of Warsaw, pp 217-229

Oberc-Dziedzic T, Kryza R (2012) Late stage Variscan magmatism in the Strzelin Massif (SW Poland): SHRIMP zircon ages of tonalite and $\mathrm{Bt}-\mathrm{Ms}$ granite of the Gęsiniec intrusion. Geol Q 56: 225-236

Oberc-Dziedzic T, Madej S (2002) The Variscan overthrust of the Lower Palaeozoic gneiss unit on the Cadomian basement in the Strzelin and Lipowe Hills massifs, ForeSudetic Block, SW Poland; is this part of the East-West Sudetes boundary? Geol Sudetica 34: 39-58

Oberc-Dziedzic T, Mades S (2013) The Skalice Crag: the migmatization of gneisses in the southern part of the Strzelin Massif. Geosci Notes 1: 53-60

Oberc-Dziedzic T, Pin C (2000) The granitoids of the Lipowe Hills (Fore-Sudetic Block) and their relationship to the Strzelin granites. Geol Sudetica 33: 17-22

Oberc J, Oberc-Dziedzic T, Klimas-August K (1988) Geological map of the Strzelin Hills $(1: 25,000)$. Institute of Geological Sciences, University of Wrocław, Geological Enterprise Wrocław (in Polish)

Oberc-Dziedzic T, Pin C, Duthou JL, Couturie JP (1996) Age and origin of the Strzelin granitoids (Fore-Sudetic Block, Poland): ${ }^{87} \mathrm{Rb} /{ }^{86} \mathrm{Sr}$ data. Neu Jb Mineral, Abh 171: $187-198$

Oberc-Dziedzic T, Żelaźniewicz A, Cwojdziński S (1999) Granitoids of the Odra Fault Zone: late- to post-orogenic Variscan intrusions in the Saxothuringian Zone, SW Poland. Geol Sudetica 32: 55-71 
Oberc-Dziedzic T, Kryza R, Klimas K, Fanning MC (2003) SHRIMP U/Pb zircon geochronology of the Strzelin gneiss, SW Poland: evidence for a Neoproterozoic thermal event in the Fore-Sudetic Block, Central European Variscides. Int J Earth Sci (Geol Rundsch) 92: 701-711

Oberc-Dziedzic T, Kryza R, Klimas K, Fanning MC, Madej $S$ (2005) Gneiss protolith ages and tectonic boundaries in the NE part of the Bohemian Massif (Fore-Sudetic Block, SW Poland). Geol Q 49: 363-378

Oberc-Dziedzic T, Kryza R, Pin C, Mochnacka K, LarioNov A (2009) The Orthogneiss and Schist Complex of the Karkonosze-Izera Massif (Sudetes, SW Poland): U-Pb SHRIMP zircon ages, Nd-isotope systematics and protoliths. Geol Sudetica 41: 3-24

Oberc-Dziedzic T, Kryza R, Białek J (2010a) Variscan multistage granitoid magmatism in Brunovistulicum: petrological and SHRIMP U/Pb zircon geochronological evidence from the southern part of the Strzelin Massif, SW Poland. Geol Q 54: 301-324

Oberc-Dziedzic T, Kryza R, Mochnacka K, Larionov A (2010b) Ordovician passive continental margin magmatism in the Central-European Variscides: U-Pb zircon data from the SE part of the Karkonosze-Izera Massif, Sudetes, SW Poland. Int J Earth Sci 99: 27-46

Oberc-Dziedzic T, Kryza R, Pin C, Madej S (2013a) Variscan granitoid plutonism in the Strzelin Massif (SW Poland): petrology and age of the Strzelin granites. Geol Q 57: 269-288

Oberc-Dziedzic T, Kryza R, Pin C, Madej S (2013b) Sequential granite emplacement: a structural study of the late Variscan Strzelin intrusion, SW Poland. Int J Earth Sci (Geol Rundsch) 102: 1289-1303

Oberc-Dziedzic T, Kryza R, Pin C (2015a) Variscan granitoids related to shear zones and faults: examples from the Central Sudetes (Bohemian Massif) and the Middle Odra Fault Zone. Int J Earth Sci (Geol Rundsch) 104: 1139-1166

Oberc-Dziedzic T, Kryza R, Pin C (2015b) Last stage of Variscan granitoid magmatism in the Strzelin Massif (SW Poland): petrology and age of the biotite-muscovite granites. Geol Q 59: 718-737

Oliver GJH, Corfu F, Krogh TE (1993) U-Pb ages from SW Poland: evidence for a Caledonian suture zone between Baltica and Gondwana. J Geol Soc, London 150: 355-369

Patchett PJ, Samson SD (2005) Ages and growth of the continental crust from radiogenic isotopes. In: RUDNICK RJ (ed) The Crust. Treatise on Geochemistry, Vol. 3. Elsevier-Pergamon, Oxford, pp 321-348

Pharaoh TC (1999) Palaeozoic terranes and their lithospheric boundaries within the Trans-European Suture Zone (TESZ): a review. Tectonophysics 314:17-41

Pietranik A, Koepke J (2009) Interactions between dioritic and granodioritic magmas in mingling zones: plagioclase record of mixing, mingling and subsolidus interactions in the Gęsiniec Intrusion, NE Bohemian Massif, SW Poland. Contrib Mineral Petrol 158: 17-36

Pietranik A, Waight TE (2008) Processes and sources during Late Variscan dioritic-tonalitic magmatism: insights from plagioclase chemistry (Gesiniec Intrusion, NE Bohemian Massif, Poland). J Petrol 49: 1619-1645

Pietranik A, Koepke J, Puziewicz J (2006) Crystallization and resorption in plutonic plagioclase: implications on the evolution of granodiorite magma (Gesiniec granodiorite, Strzelin Crystalline Massif, SW Poland). Lithos 86: $260-280$

Pietranik A, Holtz F, Koepke J, Puziewicz J (2009) Crystallization of quartz dioritic magmas at 2 and 1 kbar: experimental results. Mineral Petrol 1-2: 1-21

Pietranik A, Storey C, Dhuime B, Tyszka R, Whitehouse M (2011) Decoding whole rock, plagioclase, zircon and apatite isotopic and geochemical signatures from variably contaminated dioritic magmas. Lithos 127: 455-467

Pin C, Santos Zalduegui JF (1997) Sequential separation of light rare earth elements, thorium and uranium by miniaturized extraction chromatography: application to isotopic analyses of silicate rocks. Anal Chim Acta 339: 79-89

Rudnick RJ, GaO S (2005) Composition of the continental crust. In: Rudnick RJ (ed) The Crust. Treatise on Geochemistry, Vol. 3. Elsevier-Pergamon, Oxford, pp 1-64

Schulmann K, GaYer R (2000) A model for a continental accretionary wedge developed by oblique collision: the NE Bohemian Massif. J Geol Soc, London 157: 401-416

Suess E (1888) Das Antlitz der Erde, II. Tempsky, Wien pp 1-723

Suess FE (1912) Die moravische Fenster und ihre Beziehung zum Grundgebirge des Hohen Gesenkes. Denkschr Österr Akad Wiss Mat Naturwiss Kl 88: 541-631

SuESS FE (1926) Intrusionstektonik und Wandertektonik im variszischen Gebirge. Borntraeger, Berlin, pp 1-268

Sun SS, McDonough WF (1989) Chemical and isotopic systematics of oceanic basalts: implications for mantle composition and processes. In: SAUNDERS AD NORRY MJ (eds) Magmatism in Ocean Basins. Geological Society of London Special Publications 42: 313-345

SZCZEPAŃSKI J, OBERC-DZIEDZIC T (1998) Geochemistry of amphibolites from the Strzelin crystalline massif, Fore-Sudetic Block, SW Poland. Neu Jb Mineral, Abh 173: 23-40

Tanaka T, Togashi S, Kamioka H, Amakawa H, Kagami H, Hamamoto T, Yuhara M, Orihashi Y, Yoneda S, Shimizu H, Kunimaru T, Takahashi K, Yanagi T, Nakano T, Fuimaki H, Shinjo R, Asahara Y, Tanimizu M, Dragusanu $\mathrm{C}$ (2000) JNdi-1: a neodymium isotopic reference in consistency with LaJolla neodymium. Chem Geol 168: 279-281

Turniak K, Mazur S, Wysoczanski R (2000) SHRIMP zircon geochronology and geochemistry of the Orlica- 
Śnieżnik gneisses (Variscan belt of Central Europe) and their tectonic implications. Geodin Acta 13: 1-20

Turniak K, Tichomirowa M, Bombach K (2006) Pb-evaporation zircon ages from the Strzelin Massif (SW Poland). Miner Soc Pol Spec Pap 29: 212-215

Turniak K, Mazur S, Domańska-Siuda J, Szuszkiewicz A (2014) SHRIMP U-Pb zircon dating for granitoids from the Strzegom-Sobótka Massif, SW Poland: constrains on the initial time of Permo-Mesozoic lithosphere thinning beneath Central Europe. Lithos 208-209: 415-429

Wóscik L (1968) Detailed map of the Sudetes, map sheet Ciepłowody $1: 25,000$. Geological Institute, Wydawnictwa Geologiczne, Warsaw (in Polish)
WroŃski J (1973) Detailed map of the Sudetes, map sheet Ziębice 1:25,000. Geological Institute, Wydawnictwa Geologiczne, Warsaw (in Polish)

Žáa J, Verner K, Sláma J, Kachlík V, Chlupáčová M (2013) Multistage magma emplacement and progressive strain accumulation in the shallow-level Krkonoše-Jizera plutonic complex, Bohemian Massif. Tectonics 32: $1493-1512$

Żelaźniewicz A, Fanning CM, Achramowicz S (2009) Refining the granite, gneiss and schist interrelationships within the Lusatian-Izera Massif, West Sudetes, using SHRIMP U-Pb zircon analyses and new geologic data. Geol Sudetica 41: 67-84 